\title{
Metal Doping/Alloying of Cesium Lead Halide Perovskite Nanocrystals and their Applications in Light-Emitting Diodes with Enhanced Efficiency and Stability
}

Naresh Kumar Kumawat, Zhongcheng Yuan, Sai Bai and Feng Gao

The self-archived postprint version of this journal article is available at Linköping University Institutional Repository (DiVA):

http:// urn.kb.se/ resolve?urn=urn:nbn:se:liu:diva- 159715

N.B.: When citing this work, cite the original publication.

Kumawat, N. K., Yuan, Z., Bai, S., Gao, F., (2019), Metal Doping/ Alloying of Cesium Lead Halide Perovskite Nanocrystals and their Applications in Light-Emitting Diodes with Enhanced Efficiency and Stability, Israel J ournal of Chemistry, 59(8), 695-707. https:// doi.org/ 10.1002/ijch.201900031

Original publication available at:

https:// doi.org/ 10.1002/ijch.201900031

Copyright: Wiley-VCH Verlag (No OnlineOpen)

http:// www.wiley-vch.de/ publish/en/ 


\title{
Metal doping/alloying of cesium lead halide perovskite nanocrystals and their applications in light-emitting diodes with enhanced efficiency and stability
}

Naresh Kumar Kumawat, Zhongcheng Yuan, Sai Bai* and Feng Gao*

Department of Physics, Chemistry and Biology (IFM), Linköping University, Linköping, SE581 83, Sweden

*Email: sai.bai@liu.se, feng.gao@liu.se

\begin{abstract}
Metal halide perovskite nanocrystals (NCs) have demonstrated great advances for lightemitting diodes (LEDs) applications, owing to their excellent optical, electrical properties and cost-effective solution-processing potentials. Tremendous progress has been made in perovskite NCs-based LEDs during the past several years, with the external quantum efficiency (EQE) boosted to over 20\%. Recently, metal doping/alloying strategy has been explored to finely tune the optoelectronic properties and enhance material stability of perovskite NCs, leading to further improved device efficiency and stability of the obtained perovskite NCsbased LEDs. In this review, we summarize recent progress on the metal doping/alloying of perovskite NCs and their applications in LEDs. We focus on the effects of different metal doping strategies on the structural and optoelectronic properties of the perovskite NCs. In addition, several works on high-performance LEDs based on metal doped/alloyed perovskite NCs with different light emission colours are highlighted. Finally, we present an outlook on employing metal doping/alloying strategies to further improve the device efficiency and stability of LEDs based on perovskite NCs.
\end{abstract}

Keywords Perovskite nanocrystals, metal doping, alloying, light-emitting diodes 


\section{Biographical Information}

Dr. Naresh Kumar Kumawat is a postdoctoral at the Department of Physics, Chemistry and Biology (IFM), Linköping University (LiU). Prior to joining LiU, he received his Ph.D. from the Indian Institute of Technology Bombay (IITB), Mumbai, India. His research interests are to develop perovskite materials for blue emissive perovskite light-emitting diodes, and understand the mechanism of stability.

Mr. Zhongcheng Yuan is currently a Ph.D student in Linköping University. He received his bachelor degree (2012) and master degree (2015), majoring in materials science and engineering, in Soochow University, Suzhou, China. His research interests are now on organicinorganic hybrid perovskite light emitting diodes and solar cells.
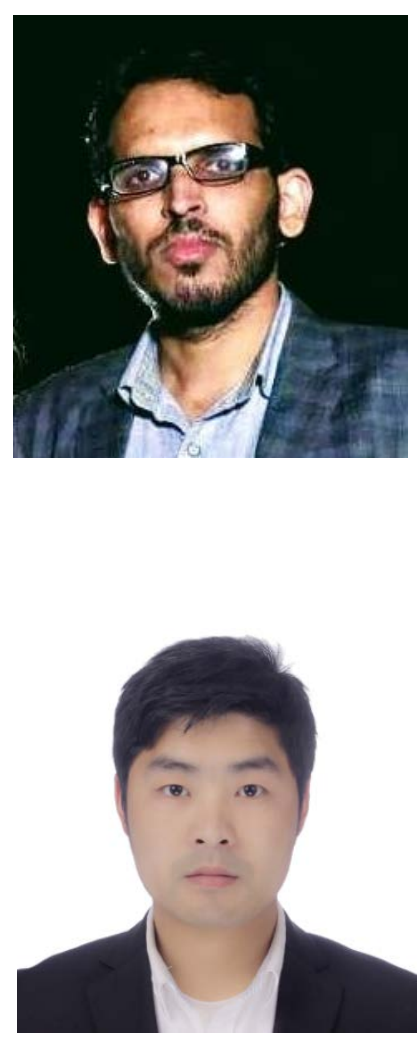

Dr. Sai Bai obtained his Ph.D. degree in Materials Physics and Chemistry from Zhejiang University in 2014, focusing on solution processable metal oxide films for optoelectronic applications. He is current an Assistant Professor in Linköping University, Sweden. His current research interests include colloidal nanocrystals, solar cells and light-emitting diodes based

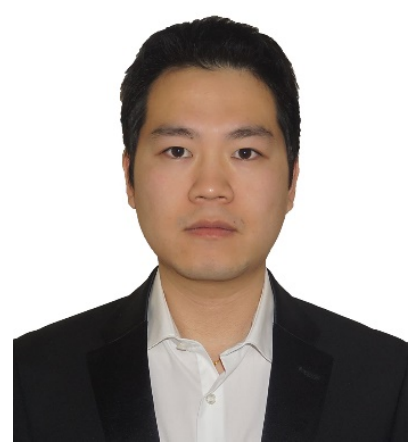
on metal halide perovskites.

Dr. Feng Gao is an Associate Professor and Wallenberg Academy Fellow at Linköping University in Sweden. He received his $\mathrm{PhD}$ from the University of Cambridge (UK) in 2011, followed by a Marie Curie postdoc fellowship at Linköping University. He received the ERC Starting Grant in 2016. His group currently focuses on the research into solution-

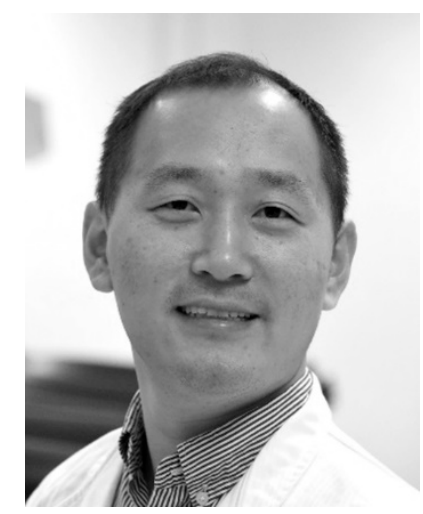


processed energy materials and devices, mainly based on organic semiconductors and metal halide perovskites.

\section{Introduction}

Solution-processed metal halide perovskites have emerged as one of the most promising semiconductors for optoelectronic devices in recent years, mainly due to their superior optoelectronic properties including widely tunable optical band gap, long and balanced charge diffusion length and high charge carrier mobility. ${ }^{[1,2,3,4,5]}$. The metal halide perovskites possess a generic formula of $\mathrm{ABX}_{3}$, where $\mathrm{A}$ is monovalent cation such as methylammonium $\left(\mathrm{MA}^{+}=\right.$ $\left.\mathrm{CH}_{3} \mathrm{NH}_{3}{ }^{+}\right)$, formamidinium $\left(\mathrm{FA}^{+}=\mathrm{CH}\left(\mathrm{NH}_{2}\right)_{2}{ }^{+}\right)$, and cesium $\left(\mathrm{Cs}^{+}\right)$; $\mathrm{B}$ is a divalent metal cation such as $\mathrm{Pb}^{2+}$ and $\mathrm{Sn}^{2+}$ ions, and $\mathrm{X}$ is a halogen anion such as $\mathrm{Cl}^{-}, \mathrm{Br}^{-}, \mathrm{I}^{-}$, which offers great opportunities to the control over the optoelectronic properties through engineering the material compositions. ${ }^{[6,7,8,9,10,11,12]}$ During the past several years, these perovskites have not only pushed forward the development of cost-effective and high-performance photovoltaic technologies but also demonstrated great advances in the applications for many other optoelectronic devices, such as light-emitting diodes (LEDs), low threshold lasing and photodetectors. ${ }^{[13,14,15,16]}$

Colloidal metal halide perovskite nanocrystals (NCs), as a newly developed type of perovskite materials, exhibit ultra-pure photoluminescence (PL) emission and super high PL quantum yield (PLQY). ${ }^{[17,18,19,20]}$ As compared to the perovskite NCs containing organic cations, the allinorganic $\mathrm{CsPbX}_{3}(\mathrm{X}=\mathrm{Cl}, \mathrm{Br}$, I or mixed $\mathrm{Cl} / \mathrm{Br}$ and $\mathrm{Br} / \mathrm{I}) \mathrm{NCs}$ are believed to be more stable due to the absence of volatile organic components in the crystal structures. ${ }^{[21,22]}$ Since the first high-quality $\mathrm{CsPbX}_{3}$ NCs reported by Kovalenko and co-workers, ${ }^{[19]}$ they have attracted increasing attention in the materials synthesis, photophysical characterizations, and optoelectronic device application. ${ }^{[23,24,25,26,27,28,29,30]}$ Several excellent reviews on perovskite 
NCs have been recently published, summarizing the chemical synthesis, material properties and applications in optoelectronic devices. ${ }^{[31,32,33,34,35]}$

As a new generation of promising semiconductor for optoelectronic applications, intense research efforts on solution engineering have been made to optimize the materials properties of perovskite NCs. ${ }^{[36,37,38]}$ For example, surface defects of the obtained perovskite NCs, which are important for the materials stability and the optoelectronic properties, can be efficiently reduced by introducing functional organic capping ligands. ${ }^{[39,40,41]}$ However, the incorporated capping ligands also affect the charge transport properties of the active layer in the resulting optoelectronic devices.$^{[42,36,37,38]}$ In addition, ligand exchange and modified purification methods were developed to improve the electrical properties of perovskite NCs, however, these approaches are likely to introduce extra surface defects, resulting in decreased PLQY and poor material stability. ${ }^{[42,43]}$ Alternatively, metal doping/alloying strategies, which are commonly used in traditional semi-conductive nanocrystals, have been successfully employed in perovskite NCs, leading to more efficient control over the optical and electronic properties of the resulting materials. ${ }^{[44,45,46,47,48]}$ At the same time, these incorporated metals ions further improve the phase, thermal, and moisture and air stability of the perovskite NCs, which could be beneficial for their wide applications in optoelectronic devices. ${ }^{[49,50]}$

Herein, we review recent progress on the metal doping/alloying of perovskite NCs, mainly focusing on the all-inorganic $\mathrm{CsPbX}_{3}$ systems, and their applications in LEDs. We start with a brief introduction of the perovskite NCs and then discuss the effects of different metal doping strategies on the structural and optoelectronic properties of the obtained perovskite NCs. In addition, we highlight several representative works on metal doping/alloying perovskite NCs and their effects on LEDs performance. Finally, we present an outlook on further exploring the metal doping/alloying strategy to improve the device efficiency and stability of perovskite NCsbased LEDs. 


\section{Chemical synthesis of colloidal $\mathrm{CsPbX}_{3} \mathrm{NCs}$}

High-crystalline monodisperse $\mathrm{CsPbX}_{3}$ nanocubes were firstly synthesized by Protesescu et al., employing a hot-injection method that was commonly used for traditional metal chalcogenide NCs (Figure 1a, 1b and 1d). ${ }^{[19]}$ The hot-injection method used for the synthesis of $\mathrm{CsPbX}_{3} \mathrm{NCs}$ involves a rapid injection of Cs precursors (e.g. Cs-oleate) into hot lead halide precursors dissolved in solvents with high boiling points (e.g. octadecene (ODE). A mixture of oleylamine and oleic acid was used to stabilize the as obtained NCs as surface ligands. The resulting $\mathrm{CsPbX}_{3}$ NCs with controllable halide compositions exhibit continuously tunable PL emission with a narrow full-width at half-maximum (FWHM) of 12-42 nm and a high PL QY of up to 90\% (Figure 1c) ${ }^{[19]}$ In addition, the perovskite NCs can also be easily synthesized using roomtemperature ligand-assisted reprecipitation (LARP) methods (Figure 1e).${ }^{[51]}$ For a typical LARP synthesis of $\mathrm{CsPbX}_{3}$ NCs, the as-prepared perovskite precursor solution in dimethylformamide/dimethyl sulfoxide (DMF/DMSO) with capping ligands, is added drop wisely into a nonpolar solvent (e.g. toluene) at room temperature. ${ }^{[51]}$ During the LARP synthesis, various type of capping ligands such as oleylamine, octylamine, oleic acid, and $\mathrm{N}$ hexadecylphosphocholine, etc. can be used to control the sizes and shapes of resulting NCs. ${ }^{[52,53]}$

In addition to the above mentioned one-pot direct synthesis methods, high-quality $\mathrm{CsPbX}_{3} \mathrm{NCs}$ with controllable halide compositions and tunable light emission can also be obtained through a post-synthetic halide anion exchange reaction. ${ }^{[54,55]}$ Nedelcu et al. ${ }^{[54]}$ and Akkerman et al. ${ }^{[55]}$ almost simultaneously reported efficient anion exchange synthesis of $\mathrm{CsPbX}_{3}$ NCs using different halide sources (Figure 1f and 1g). They observed fast reversible blue shifts or red 
shifts of the as-synthesized $\mathrm{CsPbBr}_{3} \mathrm{NCs}$ through introducing $\mathrm{Cl}^{-}$or $\mathrm{I}^{-}$sources, respectively. In addition, directly mixing perovskite NCs with different halide compositions can also initiate the anion exchange. ${ }^{[19,51]}$ The obtained perovskite NCs show minor change on the shape and crystal structure, and the PL emission of the anion-exchanged $\mathrm{CsPbX}_{3}$ NCs are similar with those directly obtained through hot-injection process, which offers a convenient approach for tuning the optoelectronic properties of perovskite NCs efficiently.
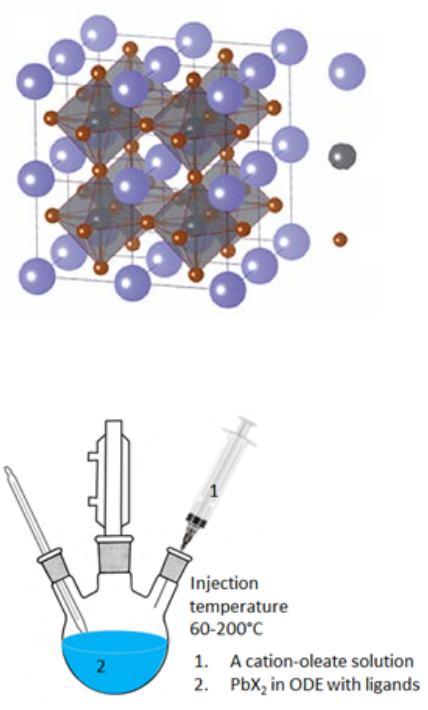

Hot Injection Method

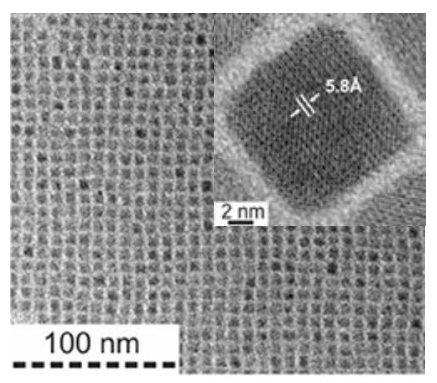

(c)

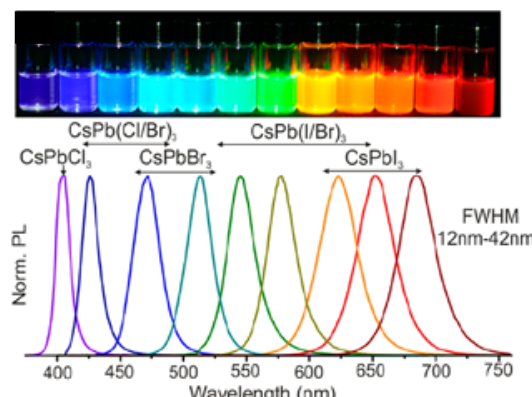

(f)

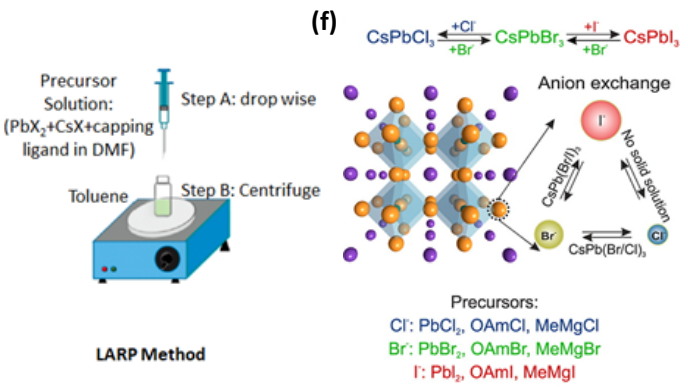

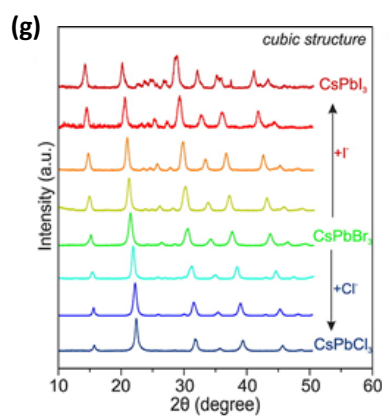

Figure 1. Structural, optoelectronic properties and chemical synthesis of perovskite NCs.

(a) Typical crystal structure of halide perovskite materials, wherein, A is a monovalent cation, $\mathrm{B}$ is a divalent metal cation, and $\mathrm{X}$ is a halogen anion. (b) Transmission electron microscopy (TEM) image of $\mathrm{CsPBr}_{3} \mathrm{NCs}$, inset image of a single NC to calculate lattice spacing. (c) Perovskite NC colloidal solution under UV irradiation (top image) and PL (bottom) spectra. Reproduced with permission. ${ }^{[19]}$ Copyright (C) 2015 American Chemical Society. (d-e) Perovskite NCs synthesis methods, hot-injection (or one-pot) and LARP. (f) Schematic representation of anion exchange approach for the synthesis of mixed halide perovskite NCs for tunable emission wavelength. (g) X-ray diffraction (XRD) pattern of perovskite NCs, which 
are synthesized by using hot-injection method via anion exchange. Reproduced with permission. ${ }^{[54]}$ Copyright (C) 2015 American Chemical Society.

\section{Metal doping/alloying of perovskite NCs}

Optoelectronic properties of perovskite NCs can be efficiently tuned through metal doping with a low concentration of metal dopants or metal alloying with a higher substitution ratio of metal elements in the perovskite crystal structure. Depending on the substitution possibility of the elements existed in the $\mathrm{ABX}_{3}$ perovskite crystal structure, we simply divide the literature reported metal doping/alloying strategies of perovskite NCs into the "A-site" and "B-site" doping/alloying. Unlike the "A-site” doping in $\mathrm{CsPbX}$ 3 perovskite NCs, of which the dopant is currently limited to monovalent Rubidium $\left(\mathrm{Rb}^{+}\right)$, various kinds of monovalent to divalent to trivalent metal ions have been efficiently incorporated into the $\mathrm{CsPbX}_{3}$ perovskite $\mathrm{NCs}$, generating interesting material properties in the resulting materials. In this section, we summarize recent works on efficient metal doping/alloying strategies with different dopants and their effects on material properties of the resulting perovskite NCs.

\subsection{A-site metal doping/alloying of perovskite NCs}

A-site doping/alloying is commonly used in previous studies on improving the material stability of perovskite films and the resulting photovoltaic devices. ${ }^{[56,57]} \mathrm{Rb}^{+}$and $\mathrm{Cs}^{+}$were intentionally added in $\mathrm{MAPbI}_{3}, \mathrm{FAPbI}_{3}$ perovskite materials to adjust the tolerance factors of the obtained perovskite structure to a more stable region and hence significantly improve the resulting film

quality and device stability. ${ }^{[56,57]}$ Although it is not quite common, A-site doping/alloying strategy was also explored in $\mathrm{CsPbX}_{3} \mathrm{NCs}$ to tune the material properties. For example, Zhao et al. reported the synthesis of $\mathrm{Rb}_{\mathrm{y}} \mathrm{Cs}_{1-\mathrm{y}} \mathrm{PbCl}_{3}$ (y is the molar concentration of doped $\mathrm{Rb}^{+}$, which is $0 \%, 3 \%, 9 \%, 15 \%)$ perovskite NCs using hot-injection method. ${ }^{[58]}$ They demonstrated partially substituted $\mathrm{Cs}^{+}$cations for $\mathrm{Rb}^{+}$, which was confirmed by the $\mathrm{XRD}$ and $\mathrm{X}$-ray 
photoelectron spectroscopy (XPS) (Figure 2a) measurements. The Rb-doped and undoped NCs show the same cubic phase as pristine $\mathrm{CsPbCl}_{3} \mathrm{NCs}$. They observed that with the increasing $\mathrm{Rb}^{+}$concentration, the main diffraction peaks in the XRD shifted towards higher diffraction angle, indicating possible lattice contraction after incorporation of $\mathrm{Rb}^{+}$. They further confirmed that the lattice space decreased from $3.96 \AA$ to $3.95 \AA$ through high resolution transmission electron microscopy (HRTEM) characterizations of the undoped (Figure 2b) and doped (Figure 2c) NCs.

Amgar et al. also demonstrated shifted XRD peaks toward higher diffraction angle in the $\mathrm{Rb}^{+}$ doped $\mathrm{CsPbCl}_{3}$ and $\mathrm{CsPbBr}_{3}$ NCs with increasing $\mathrm{Rb}^{+}$concentration. ${ }^{[59]}$ The XRD diffraction peaks are identical to $\mathrm{CsPbCl}_{3}$ perovskite when $x=0.4$ (here, $x$ is $\mathrm{Rb}^{+}$concentration), while new peaks belonging to $\mathrm{RbPb}_{2} \mathrm{Cl}_{5}$ phase appeared when $x$ is higher than 0.4 (Figure $2 \mathrm{~d}$ ). They observed blue shift in the band edge of $\mathrm{CsPbCl}_{3} \mathrm{NCs}$ with higher $\mathrm{Rb}^{+}$concentration (Figure 2e), ascribing to the lattice contraction due to a smaller size of $\mathrm{Rb}^{+}(174 \mathrm{pm})$ as compared to that of $\mathrm{Cs}^{+}(161 \mathrm{pm})$. With the optimal $\mathrm{Rb}^{+}$incorporation ratio, PLQY of the resulting greenand blue-emissive NCs increased to over $60 \%$ and $12 \%$, respectively (Figure $2 \mathrm{f}$ ). In contrast to the claimed more stable lattice structure of the $\mathrm{Rb}^{+}$doped $\mathrm{CsPbX}$ NCs, Linaburg et al. performed theoretical simulation and concluded that the $\mathrm{Rb}^{+}$doped perovskites are likely to form unstable crystal structure. ${ }^{[60]}$ Considering the very small difference in the HRTEM and the XRD results, further investigation on the change to the crystal lattice and the materials stability of $\mathrm{Rb} / \mathrm{CsPbX} 3$ NCs are still needed. 

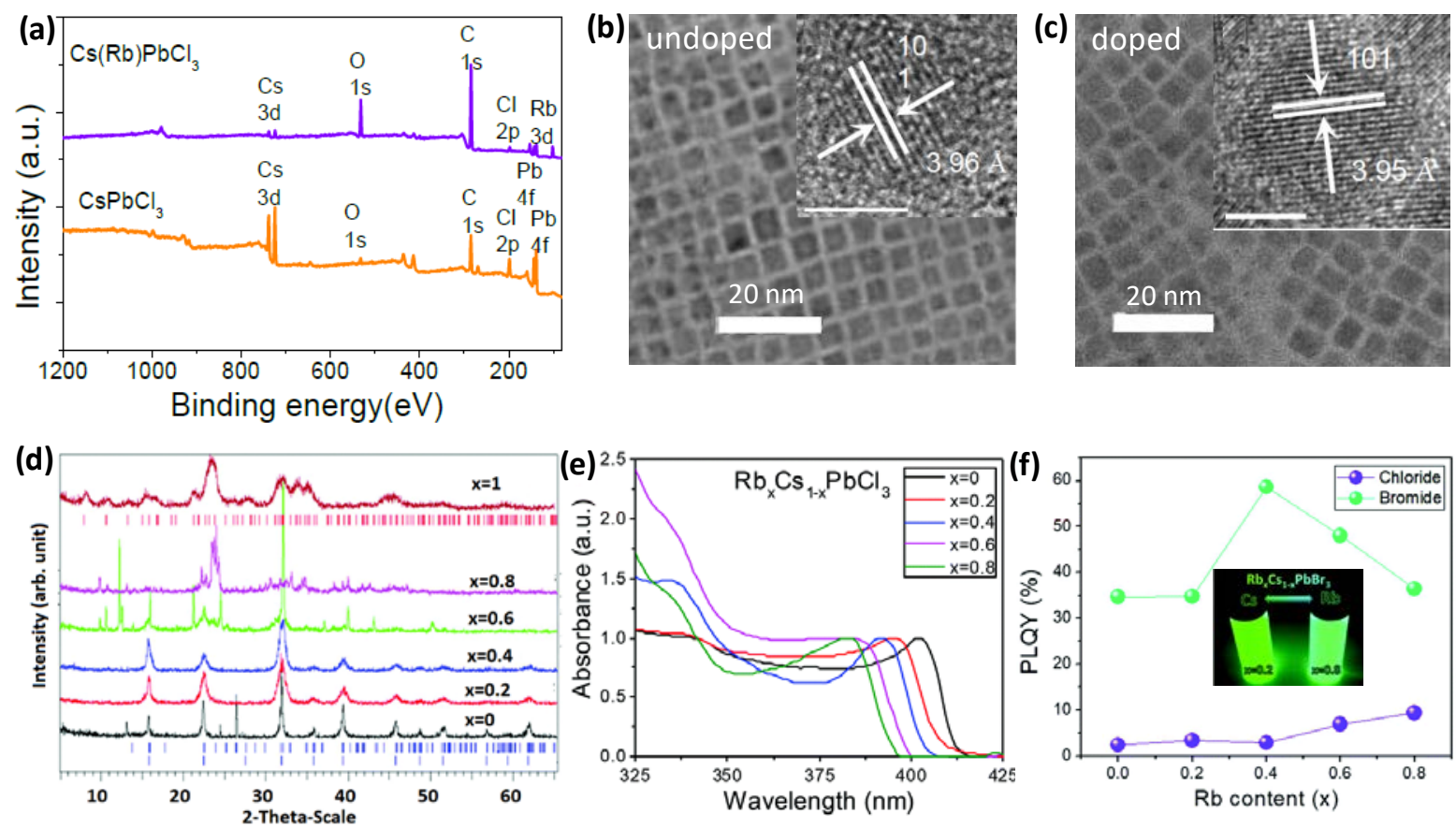

Figure $2 \mathbf{R b}^{+}$doped PNCs and their optoelectronic properties: (a) XPS spectra of pure $\mathrm{CsPbCl}_{3} \mathrm{NCs}$ and $\mathrm{CsPbCl}_{3}: 9 \%$ doped NCs. (b-c) TEM images of undoped and doped perovskite NCs, the inset image of a single perovskite NC. Reproduced with permission. ${ }^{[58]}$ copyright (C 2018 Elsevier Ltd. (d) XRD pattern of metal doped $\mathrm{CsPbCl}_{3}$ perovskite NCs with $\mathrm{Rb}^{+}$concentration. (e) Absorbance spectra of $\mathrm{CsPbCl}_{3} \mathrm{NCs}$ with $\mathrm{Rb}^{+}$doping concentration. (f) PLQY of $\mathrm{CsPbBr}_{3}$ and $\mathrm{CsPbCl}_{3}$ perovskite NCs with $\mathrm{Rb}^{+}$concentration, the inset images of undoped (left) and doped (right) $\mathrm{CsPbBr}_{3}$ perovskite $\mathrm{NC}$ colloidal solution under UV irradiation. Reproduced with permission. ${ }^{[59]}$ copyright $@$ The Royal Society of Chemistry 2018.

\subsection{B-site metal doping/alloying of perovskite NCs}

\subsubsection{Isovalent B-site doping/alloying of perovskite NCs with $\mathrm{Mn}^{2+}$ ions}

As an efficient doping strategy in tuning the material properties of traditional quantum dots (QDs), doping of transition metal of $\mathrm{Mn}^{2+}$ in $\mathrm{CsPbX}_{3}$ perovskite NCs have drawn intense attention since two pioneering reports by Parobek et al. ${ }^{[44]}$ and Liu et al. in $2016 .{ }^{[45]}$ In the two 
works, $\mathrm{CsPbCl}_{3} \mathrm{NCs}$ with low $\mathrm{Mn}^{2+}$ doping concentration were synthesized following the welldeveloped one-pot hot injection synthesis. $\mathrm{MnCl}_{2}$ was used to control the incorporated $\mathrm{Mn}^{2+}$ concentration during the synthesis. Liu et al. carefully investigated the importance of the precursor for the $\mathrm{Mn}^{2+}$ doping and did not observe any doping if they substitute the manganese chloride $\left(\mathrm{MnCl}_{2}\right)$ for manganese acetate $\left(\mathrm{Mn}(\mathrm{ac})_{2}\right)$, manganese acetylacetonate $\left(\mathrm{Mn}(\mathrm{acac})_{2}\right)$, or manganese oleate ( $\mathrm{Mn}(\text { oleate) })_{2}$ ), demonstrating the benefits of the $\mathrm{Mn}-\mathrm{Cl}$ bond and an allCl coordination environment for efficient $\mathrm{Mn}^{2+}$ doping. ${ }^{[45]}$ The obtained highly crystalline $\mathrm{Mn}-$ doped $\mathrm{CsPCl}_{3}$ perovskite NCs show nearly identical orthorhombic phase (Figure 3a and 3b), similar cubic shape and size compared with the undoped ones. Both works on Mn-doped perovskite NCs demonstrated minor changes to the absorption spectra, but an additional broad PL peak at $\sim 600 \mathrm{~nm}$ (Figure 3c) attributed $\mathrm{Mn}^{2+} \mathrm{d}-\mathrm{d}$ emission appeared in the obtained Mndoped $\mathrm{CsPCl}_{3} \mathrm{NCs}$ with low doping ratio. Monotonically shifted peaks to higher angles with increasing $\mathrm{Mn}^{2+}$ doping concentration (Figure 3d and 3e), which is ascribed to the incorporated relatively smaller size of $\mathrm{Mn}^{2+}$ ions (97 pm) as compared with $\mathrm{Pb}^{2+}$ ions (133 pm). It was also demonstrated that the $\mathrm{Mn}^{2+}$ doping can obviously improve the overall PLQYs to $~ 60 \%$.

The efficient $\mathrm{Mn}^{2+}$ doping was further successfully explored to the preparation of perovskite NCs with mixed halide compositions (Figure 3f), either from the directly hot-injection or postsynthetic anion exchange methods. Interestingly, an obvious decrease in the dopant PL emission were observed for Mn-doped mixed halide perovskite NCs obtained from the anion exchange method. Gamelin and co-workers found that the PL and PL decay of Mn-doped $\mathrm{CsPbCl}_{3} \mathrm{NCs}$ exhibit an unusual temperature dependence, which implies a combination of the distinct thermal characteristics of excitons in $\mathrm{CsPCl}_{3} \mathrm{NCs}$ and the slow energy transfer to $\mathrm{Mn}^{2+}$ dopant in the doped NCs. ${ }^{[61]}$ It was also found that higher dopant concentrations above $\sim 3 \%$ led to reduced PLQYs of the resulting Mn-doped NCs, which was ascribed to the formation of $\mathrm{Mn}^{2+}-\mathrm{Mn}^{2+}$ 
pairs and the introduction of new non-radiative recombination pathways under high doping concentration.

$\mathrm{CsPbX}_{3} \mathrm{NCs}$ with elevated $\mathrm{Mn}^{2+}$ substitution ratio was further demonstrated through modifying the material synthesis process. $\mathrm{Li}$ and co-workers reported that the $\mathrm{Mn}^{2+}$ concentration can reach a maximum atomic percentage of over 37\% through the one-pot synthesis via roomtemperature supersaturated crystallization method. ${ }^{[62]}$ Enhanced broad dopant emission with the higher substitution $\mathrm{Mn}^{2+}$ ratio was observed. Through employing a phosphine-free hotinjection synthesis, the replacement ratio of $\mathrm{Pb}^{2+}$ with $\mathrm{Mn}^{2+}$ can be further increased to $\sim 46 \%$. ${ }^{[46]}$ Yang and co-workers found that the as-prepared $\mathrm{CsPbCl}_{3} \mathrm{NCs}_{\text {can }}$ still retain the tetragonal crystalline structure under such high $\mathrm{Mn}^{2+}$ substitution ratio. ${ }^{[46]}$ A further improved PLQY of $54 \%$ was achieved for the $\mathrm{CsPb}_{0.73} \mathrm{Mn}_{0.27} \mathrm{Cl}_{3}$ NCs synthesized. It was believed that the muchenhanced dopant emission is because of to the efficient energy transfer of photoinduced excitons from the $\mathrm{CsPCl}_{3}$ host to the $\mathrm{Mn}^{2+}$ dopant, which facilitates the radiative exciton recombination. Further increasing the $\mathrm{Mn}^{2+}$ substitution ratio reduces the crystallinity of the obtained NCs and decreases the PLQYs. ${ }^{[63]}$ The dopant emission in $\mathrm{Mn}$-doped $\mathrm{CsPbCl}_{3}$ was demonstrated to be tunable with the introduction of alkylamine hydrochloride $\left(\mathrm{RNH}_{3} \mathrm{Cl}\right) .{ }^{[63]}$ Pradhan and co-workers found that the $\mathrm{RNH}_{3} \mathrm{Cl}$ is helpful to create more particles with smaller dimension, which resulted in more efficient $\mathrm{Mn}^{2+}$ doping. ${ }^{[63]}$ With this optimized material synthesis, further enhanced dopant emission and minimal excitonic emission from the host $\mathrm{CsPbCl}_{3} \mathrm{NCs}$ through optimization of the material synthesis process were obtained. ${ }^{[63]}$ 

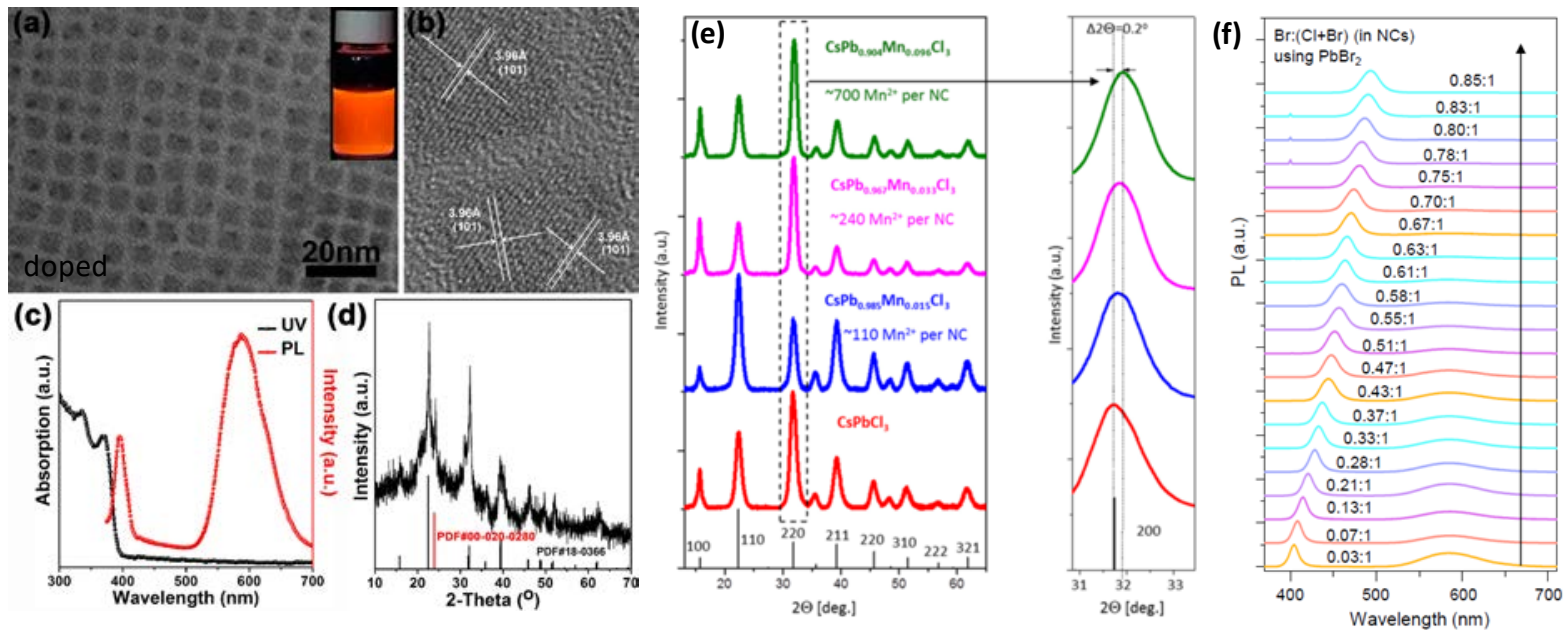

Figure 3. Mn-doped perovskite NCs and their material properties: (a-b) TEM and HRTEM image of doped $\mathrm{CsPbCl}_{3} \mathrm{NCs}$, the inset image of doped perovskite $\mathrm{NC}$ colloidal solution under UV irradiation. (c-d) Absorbance spectra and XRD pattern of $\mathrm{Mn}^{2+}$ doped $\mathrm{CsPbCl}_{3}$ perovskite NCs. Reproduced with permission. ${ }^{[46]}$ Copyright (C) 2017, American Chemical Society. (e) XRD pattern of $\mathrm{CsPbl}_{3}$ perovskite NCs with Mn doping concentration. (f) PL spectra of Mn doped $\mathrm{CsPbCl}_{3}$ perovskite $\mathrm{NCs}$ with halide ion ratio to eliminate the $600 \mathrm{~nm}$ emission band. Reproduced with permission. ${ }^{[45]}$ Copyright $(C)$ 2016, American Chemical Society.

\subsubsection{Isovalent B-site doping/alloying of perovskite $\mathrm{NCs}$ with $\mathrm{Zn}^{2+}, \mathrm{Cd}^{2+}, \mathrm{Sn}^{2+}, \mathrm{Cu}^{2+}, \mathrm{Ni}^{2+}$ ions}

Apart from the mentioned $\mathrm{Mn}^{2+}$ doping for $\mathrm{CsPbX}_{3} \mathrm{NCs}$, several other divalent metal ions were also reported to replace $\mathrm{Pb}^{2+}$ and achieve efficient doping of $\mathrm{CsPbX}_{3} \mathrm{NCs}$. Stam et al. first demonstrated the $\mathrm{Sn}^{2+}, \mathrm{Cd}^{2+}$ and $\mathrm{Zn}^{2+}$ doping of $\mathrm{CsPbBr}_{3}$ NCs using post-synthetic cation exchange reactions using the $\mathrm{SnBr}_{2}, \mathrm{CdBr}_{2}$, and $\mathrm{ZnBr}_{2}$ as the metal ion sources. ${ }^{[64]}$ They observed obvious blue-shift in the absorption and PL emission spectra after doping (Figure 4a and 4b), while high PLQYs (over 50\%) and narrow PL emission of the parent $\mathrm{CsPbBr}_{3} \mathrm{NCs}$ were preserved. The observed blue-shift in the optical spectra of metal doped $\mathrm{CsPbBr}_{3} \mathrm{NCs}$ was attributed to a possible lattice contraction of the perovskite unit cells, which causes shorter 
$\mathrm{Pb}-\mathrm{Br}$ bonds and a stronger ligand field within the Pb-halide octahedra. Considering the reported low PLQYs and broad PL emission spectra of blue-emitting $\mathrm{CsPbCl}_{3} \mathrm{NCs}_{\text {obtained }}$ from direct hot-injection or anion exchange approaches, metal doping of $\mathrm{CsPbBr}_{3} \mathrm{NCs}$ may offer new possibilities for the synthesis of high-quality perovskite NCs for blue-emitting LEDs. Similarly, Shen et al. synthesized high-quality $\mathrm{Zn}^{2+}$ alloyed $\mathrm{CsPbI}_{3} \mathrm{NCs}$, which exhibit similar blue-shift phenomenon and a super high PLQY of 98.5\% (Figure 4c and Figure 4d) with an optimal doping ratio of $0.35 \%$ for $\mathrm{Zn}$-alloyed $\mathrm{CsPbI}_{3} \mathrm{NCs}$ due to the suppressed non-radiative recombination. Further increasing the alloying ratio to 0.49 resulted in a decreased PLQY to $78 \%$, which was due to the increased defect density or a possible increase in the dark fraction of emitters at higher Zn contents. ${ }^{[65]}$

In a recent work, Li et al. employed a post-treatment method of the perovskite NCs employing Zn halide salt/hexane solution achieved significantly increased PLQY of the $\mathrm{CsPbX}_{3} \mathrm{NCs}$, boosting the optimum PLQYs to 86, 93, and 95\% for $\mathrm{CsPbCl}_{3}, \mathrm{CsPbBr}_{3}$ and $\mathrm{CsPbI}_{3} \mathrm{NCs}$, respectively. ${ }^{[66]}$ Interestingly, unlike the previous demonstrated $\mathrm{Zn}^{2+}$ doping, which exhibited obvious blue-shift of the PL emission, no change in the PL emission peaks were observed, which suggested that the post treatment might only affect the surface of the perovskite NCs. The obviously increased PLQYs were also believed to be associated with the effectively suppressed non-radiative recombination pathways, as a result of the decreased charge trap states at the surface of NCs after post treatment with Zn halide salt/hexane solution.

Following the divalent ion doping strategy, Mondal et al. also introduced $\mathrm{Cd}^{2+}$ ion and demonstrated a significantly enhance PLQY 96\% for blue-violet-emitting $\mathrm{CsPbCl}_{3} \mathrm{NCs}$ without changing the PL peak position at $406 \mathrm{~nm}$ and the spectral FWHM. ${ }^{[67]}$ Further characterizations on the stability of Cd-doped NCs showed almost no changes in the PL emission for 2 months in dark conditions. In addition, the obtained Cd-doped NCs also exhibit excellent photostability. No change in the PL peak emission was observed and $60 \%$ of the initial 
PLQY can be retained after 260 h continuous illumination under 365 nm UV light. Bi et. al demonstrated an efficient $\mathrm{Cu}^{2+}$ doping of $\mathrm{CsPbX}_{3}$ in improving the thermal stability and optoelectronic properties of the resulting materials. High PLQYs of 95\% and 80\% for greenemissive $\mathrm{CsPb}_{1-\mathrm{x}} \mathrm{Cu}_{\mathrm{x}} \mathrm{Br}_{3}$ and blue-emissive $\mathrm{CsPb}_{1-\mathrm{x}} \mathrm{Cu}_{\mathrm{x}}(\mathrm{Br} / \mathrm{Cl})_{3}$ NCs were successfully demonstrated, respectively. ${ }^{[68]}$ In addition, $\mathrm{Ni}^{2+}$ ions were also obtained to be effective in improving the optical properties of the $\mathrm{CsPbX}_{3} \mathrm{NCs}$, leading to a strong single-colour violet emission with a high PLQY of 96.5\% (Figure 4e). ${ }^{[69]}$ The doping of $\mathrm{Ni}^{2+}$ can efficiently remove the structural defects i.e. halide ion vacancies, resulting in improved short-range lattice order and improved radiative recombination of the perovskite NCs (Figure 4f). Similar improvements on the PLQYs of perovskite NCs with different $\mathrm{Br} / \mathrm{Cl}$ composition were also demonstrated, further demonstrating the potential of using metal doping/alloying strategy on the synthesis of highly emissive perovskite NCs for efficient blue-emitting LEDs.
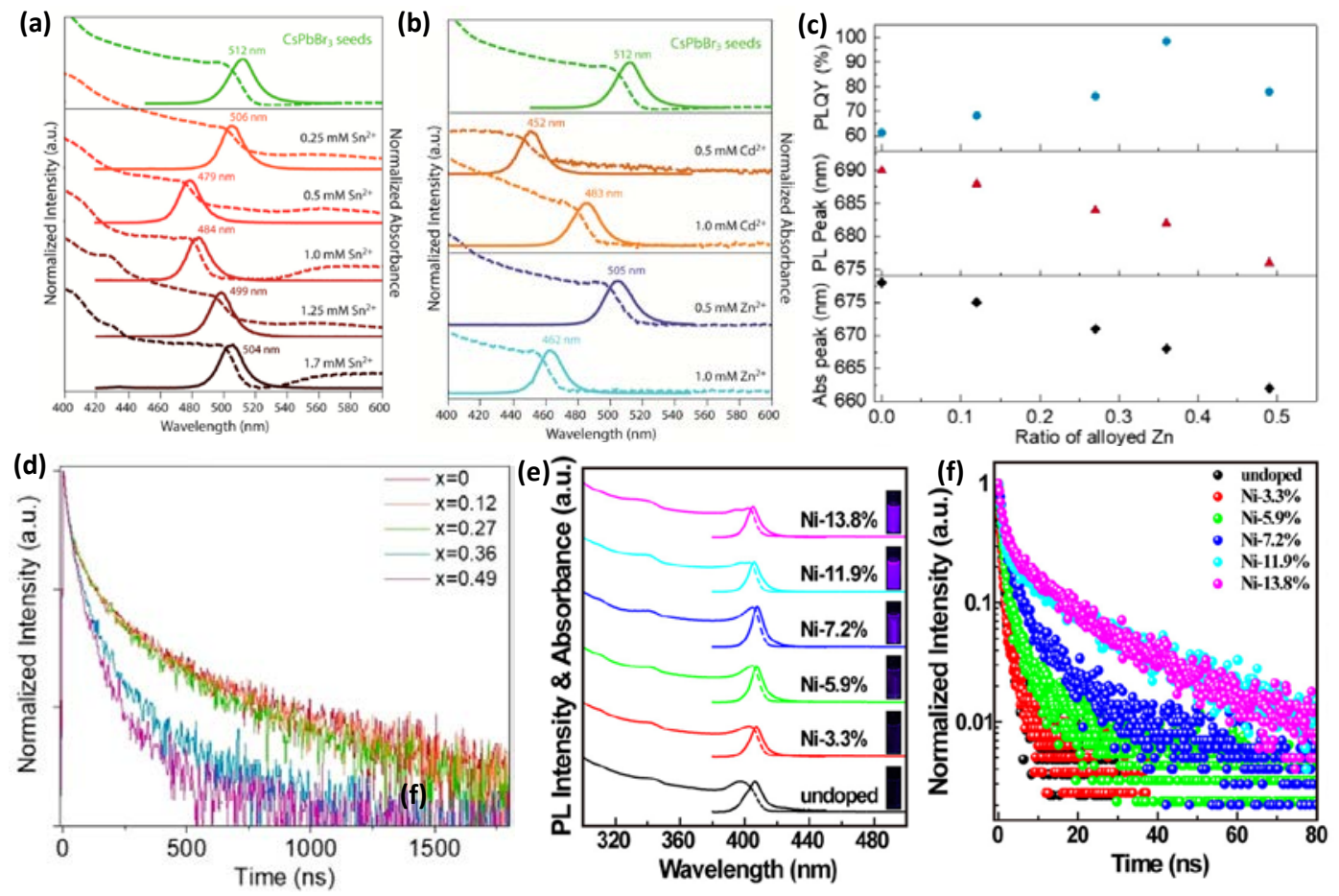

Figure 4. Optoelectronic properties of $\mathrm{Zn}^{2+}, \mathrm{Cd}^{2+}, \mathrm{Sn}^{2+}$, and $\mathrm{Ni}^{2+}$ doped perovskite NCs: (a-b) Absorption and PL spectra of $\mathrm{Sn}^{2+}, \mathrm{Cd}^{2+}$, and $\mathrm{Zn}^{2+}$ doped $\mathrm{CsPbBr}_{3}$ perovskite NCs. 
Reproduced with permission. ${ }^{[64]}$ Copyright (C) 2017 American Chemical Society. (c) PL QYs, PL peaks and absorbance peaks of $\mathrm{Zn}^{2+}$ doped $\mathrm{CsPbI}_{3}$ perovskite NC. (d) PL decay life time of $\mathrm{CsPbI}_{3} \mathrm{NCs}$ with $\mathrm{Zn}^{2+}$ metal doping concentration. Reproduced with permission. ${ }^{[65]}$ Copyright (C) 2019 American Chemical Society. (e) Absorbance and PL spectra of $\mathrm{CsPbCl}_{3} \mathrm{NC}$ with $\mathrm{Ni}^{2+}$ doping concentration, the inset images are of NC colloidal solution under UV irradiation (f) PL decay of $\mathrm{Ni}^{2+}$ doped $\mathrm{CsPbCl}_{3}$ NC. Reproduced with permission. ${ }^{[69]}$ Copyright $(\mathrm{C} 2018$ American Chemical Society.

\subsubsection{Heterovalent B-site metal doping/alloying of perovskite NCs}

In addition to the isovalent doping of perovskite NCs using transition metal ions, doping of $\mathrm{CsPbX} 3$ NCs using heterovalent ions such as $\mathrm{Ag}^{+}, \mathrm{Al}^{3+}, \mathrm{Bi}^{3+}$ or various lanthanide ions were also frequently reported. ${ }^{[70,71,72,73]}$ The possibility of heterovalent doping of perovskite NCs was first demonstrated by Mohammed and co-workers with the incorporation of $\mathrm{Bi}^{3+}$ in $\mathrm{CsPbBr}_{3} \mathrm{NCs}$ (Figure $5 \mathrm{a}$ and $5 \mathrm{~b}$ ). ${ }^{[72]}$ The successful incorporation of $\mathrm{Bi}^{3+}$ into the NCs was confirmed through the XPS and optical characterization results. They observed clearly increased $\mathrm{Bi} / \mathrm{Pb}$ ratios with increasing amount of $\mathrm{BiBr}_{3}$ in the feed solution even after several times of washing of the NCs. The excitonic peak first showed redshift under a lower $\mathrm{Bi}^{3+}$ doping ratio of $0.25 \%$ while a blueshift appeared when the doping ratio increased to $0.8 \%$ (Figure $5 \mathrm{c}$ and Figure 5d), further indicating successful incorporation of $\mathrm{Bi}^{3+}$ into the NCs. In a similar work, Zhu et al. also investigated the material properties of $\mathrm{Bi}^{3+}$ doped $\mathrm{CsPbI}_{3} \mathrm{NCs}^{[74]}$ They observed that the emission colour of the NC solution changed from red to brown with increasing the $\mathrm{Bi}^{3+}$ doping concentration. Interestingly, although they noticed decreased band-edge PL emission at $680 \mathrm{~nm}$ with increasing $\mathrm{Bi}^{3+}$ doping concentration, an ultrawide near infrared (NIR) emission from 800 to $1600 \mathrm{~nm}$ peaked at $1145 \mathrm{~nm}$ were also detected in Bi-doped $\mathrm{CsPbI}_{3} \mathrm{NCs}$, while no such NIR emission appeared in undoped parent NCs. The wide NIR emission was ascribed to the introduced Bi-related NIR active centers in the doped NCs. 
More obvious optical shift in the absorption and PL emission spectra was demonstrated through a similar heterovalent doping of $\mathrm{CsPBBr}_{3} \mathrm{NCs}$ with $\mathrm{Al}^{3+}$ ions. ${ }^{[1]}$ The incorporation of $\mathrm{Al}^{3+}$ ions resulted in an obvious blue shift of $\sim 50 \mathrm{~nm}$ in both the absorption and emission spectra (Figure 5e and 5f) of the resulting perovskite NCs. Based on the calculation results, the $\mathrm{Al}^{3+}$ doping may result in a new energy level in the bandgap of the NCs, due to the hybridization of the $\mathrm{Al}$ s-orbitals, the $\mathrm{Br}$ p-orbitals, and the $\mathrm{Pb}$ p-orbitals. As a result, the introduced contraction of the $\mathrm{PbBr}_{6}$ octahedral led to shorter $\mathrm{Pb}-\mathrm{Br}$ bonds and stronger interactions, which further improved the thermal stability of $\mathrm{Al}$-doped $\mathrm{CsPbBr}_{3} \mathrm{NCs}$ film. Almost no change was observed in the PL emission of blue $\mathrm{Al}$-doped $\mathrm{CsPbr}_{3}$ films under elevated temperature of $100{ }^{\circ} \mathrm{C}$ and thermal recycling, while the relative PL intensity of undoped NCs decreased to $60 \%$ after the heat treatment. $^{[71]}$

The possibility of introducing monovalent $\mathrm{Ag}^{+}$doping into $\mathrm{CsPb}_{3}$ perovskite $\mathrm{NCs}$ was first reported on Ag substrates-based LEDs by Rogach and co-workers. ${ }^{[70]}$ The existence of $\mathrm{Ag}^{+}$in the perovskite NCs was confirmed by the elemental mapping and the XPS characterizations. They also found that the $\mathrm{Ag}^{+}$doping efficiently passivated the surface defect states of $\mathrm{CsPbI}_{3}$ NCs, leading to improvement of PLQY and material stability. A controllable doping of $\mathrm{Ag}^{+}$ into $\mathrm{CsPBr}_{3} \mathrm{NCs}$ was then achieved by Zhou et al. via a room-temperature synthesis with the $\mathrm{Ag}_{2} \mathrm{CO}_{3}$ as the precursor. ${ }^{[75]}$ Both undoped and Ag-doped $\mathrm{CsPbBr}_{3}$ NCs exhibited an orthorhombic crystal structure and show no obvious change in the size of NCs. A tiny red-shift in the PL emission from 512 to 519 nm was observed, together with obviously quenched PL emission, which is consistent with increased trap states in the obtained Ag-doped $\mathrm{CsPbBr} 3 \mathrm{NCs}$, similar to that of $\mathrm{Bi}^{3+}$ doping in $\mathrm{CsPbBr}_{3} \mathrm{NCs}{ }^{[72]}$ Interestingly, the $\mathrm{Ag}^{+}$doping caused a heavy p-type character in the obtained NCs, with the Fermi level down toward the valence band with increasing Ag doping concentration to $0.48 \%{ }^{[75]}$ In addition, the $\mathrm{Ag}^{+}$doping also introduced considerable improvements in the film conductivity and hole mobility based on the field-effect- 
transistors characterizations results. Nearly 3 orders of magnitude improvement in the hole mobility at room temperature compared with the undoped $\mathrm{CsPbBr} 3 \mathrm{NCs}_{3}$ was demonstrated. ${ }^{[75]}$ Multicolor emissions of $\mathrm{CsPbX}_{3}$ NCs spanning from visible to NIR regions were successfully obtained by Pan et al., through introducing efficient doping of various lanthanide ions (Cerium $\left(\mathrm{Ce}^{3+}\right)$, Samarium $\left(\mathrm{Sm}^{3+}\right)$, Europium $\left(\mathrm{Eu}^{3+}\right)$, Terbium $\left(\mathrm{Tb}^{3+}\right)$, Dysprosium $\left(\mathrm{Dy}^{3+}\right)$, Erbium $\left(\mathrm{Er}^{3+}\right)$, and Ytterbium $\left.\left(\mathrm{Yb}^{3+}\right)\right)$ (Figure $5 \mathrm{~g}$ and $\left.5 \mathrm{~h}\right) .{ }^{[76]}$ They found that the lanthanide ions doping not only led to bright and abundant emissions from the dopants, but also considerably improved overall PLQYs of the host $\mathrm{CsPbCl}_{3} \mathrm{NCs}$. Incorporation of the lanthanide dopants into the perovskite NCs was confirmed based on the theoretical calculation, the XPS and inductively coupled plasma mass spectrometry (ICP-MS) characterization results. The enlarged band gap of the NC host and the blue shift in the band-edge emission with increasing the doped ions further confirmed the lanthanide doping into $\mathrm{CsPbCl}_{3} \mathrm{NCs}$. At the meantime, several intense PL emission peaks origin from the intrinsic electronic transitions of lanthanide ions appeared in the lanthanide ions doped NCs, which suggested an efficient energy transfer from the host $\mathrm{CsPbCl}_{3} \mathrm{NCs}$ to the energy levels of lanthanide ions. It was also demonstrated that the doping of lanthanide ions introduced an obvious enhancement of the overall PLQYs of the $\mathrm{CsPbCl}_{3}$ NCs, possibly due to the modification of the defects and the reduced non-radiative recombination pathways. In addition, co-doping of the perovskite NCs employing lanthanide ions of $\mathrm{Yb}^{3+} / \mathrm{Ce}^{3+}$ or $\mathrm{Yb}^{3+} / \mathrm{Er}^{3+}$ were also investigated. ${ }^{[77]}$ Strong emission of $\mathrm{Yb}^{3+}$ at $988 \mathrm{~nm}$ was demonstrated and a high overall PLQY approaching 146\% can be obtained in the $\mathrm{Yb}^{3+} / \mathrm{Ce}^{3+}$ co-doped $\mathrm{CsPbCl}_{1.5} \mathrm{Br}_{1.5} \mathrm{NCs}$, which was successfully employed on commercial silicon solar cells and exhibited an efficiency improvement from $18.1 \%$ to $21.5 \%{ }^{[77]}$

The photophysical properties of lanthanide $\mathrm{Yb}^{3+}$ ions doped $\mathrm{CsPbCl}_{3}$ NCs were carefully investigated by Gamelin and co-workers. ${ }^{[78]}$ High-quality undoped and doped $\mathrm{CsPbCl}_{3} \mathrm{NCs}$ were synthesized through the traditional hot-injection methods through introducing metal- 
acetate salts and chlorotrimethysilane as the cation and halide precursors, respectively. Intense NIR PL emission centered at $990 \mathrm{~nm}$, which is attributed to $\mathrm{Yb}^{3+}$ dopant emission, was observed in the doped NCs. The excitonic PLQYs of the undoped samples decreased sharply from 20\% to less than $1 \%$ after the incorporation of $\mathrm{Yb}^{3+}$ dopants at a low concentration to $\sim 0.7 \%$. They demonstrated that, in contrast to the optical characteristics of previous reported $\mathrm{Mn}^{2+}$ doping $\mathrm{CsPbCl}_{3} \mathrm{NCs}$, energy transfer from the $\mathrm{NCs}$ to $\mathrm{Yb}^{3+}$ was not suppressed at cryogenic temperature, which is consistent with the observation that the energy transfer is fast enough to compete with the exciton recombination. Replicable high PLQYs exceeding $100 \%$, were measured in the $\mathrm{Yb}^{3+}$ doped perovskite NCs. It was demonstrated that the extremely high PLQYs may result from extremely fast energy transfer from the energy captured by an $\mathrm{Yb}^{3+}$ induced defects to two neighboring $\mathrm{Yb}^{3+}$ ions in a single concerted step in picoseconds.
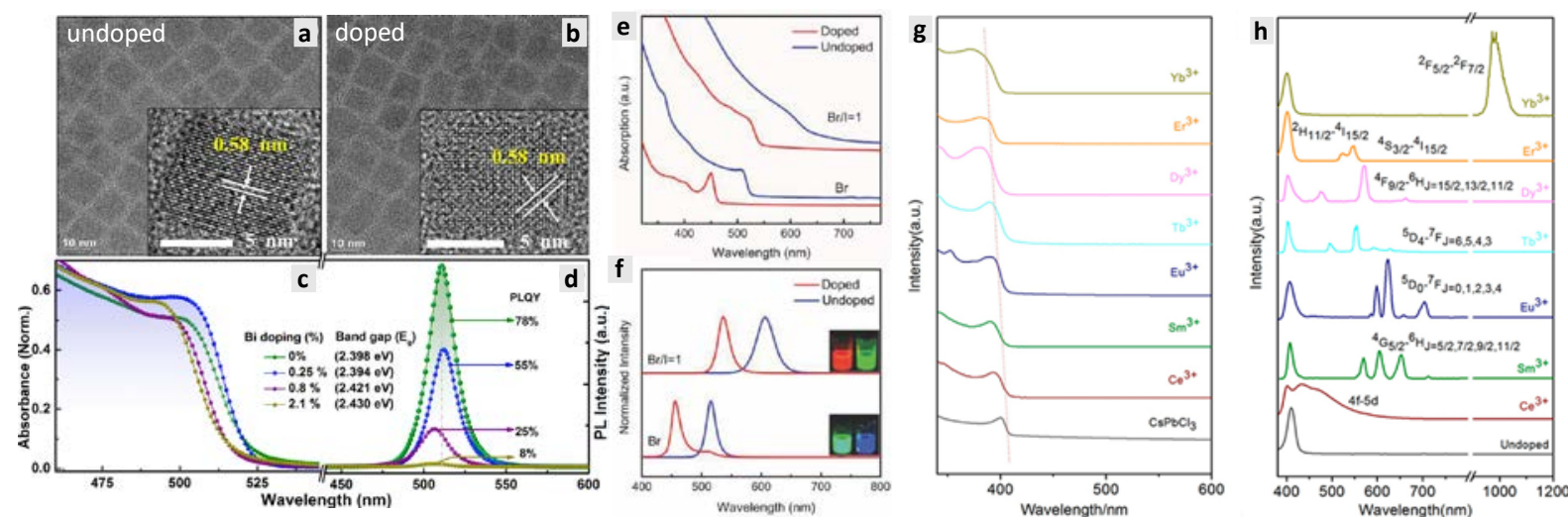

Figure 5. (a-b) TEM image of undoped and $\mathrm{Bi}$-doped $\mathrm{CsPbBr}_{3} \mathrm{NCs}$, inset image of single NC (c) Absorbance and (d) PL spectra of $\mathrm{CsPbBr}_{3} \mathrm{NCs}_{\text {with }} \mathrm{Bi}^{3+}$ doping concentration. Reproduced with permission. ${ }^{[72]}$ Copyright (c) 2016 American Chemical Society. (e-f) Absorbance and PL spectra of $\mathrm{Al}$-doped $\mathrm{CsPbI}_{1.5} \mathrm{Br}_{1.5}$ and $\mathrm{CsPbBr}_{3}$ NCs. Reproduced with permission. ${ }^{[71]}$ (g-h) Absorption and PL spectra of lanthanide doped $\mathrm{CsPbCl}_{3}$ NCs. Reproduced with permission. ${ }^{[76]}$ Copyright (C) 2017, American Chemical Society. 


\section{High-performance LEDs based on perovskite NCs with metal doping/alloying}

LEDs based on perovskite NCs have attracted booming attentions during last several years, with the device EQE has rapidly reached to over $20 \%$ within four years, well comparable to the conventional LEDs fabricated from organic semiconductors and quantum dots. ${ }^{[79,80,81]}$ However, the moderate material and device stability are still main concerns of state-of-the-art LEDs based on perovskite NCs for practical applications. ${ }^{[79,82]}$ The developed controllable metal doping/alloying strategies are effective in controlling the optical, electronic properties as well as improving the material stability of perovskite NCs, offering new possibilities for the enhancement of the device performance of the resulting LEDs. In this section, we briefly summarize several representative works on LEDs with different emission colours based on metal doped/alloyed all-inorganic $\mathrm{CsPbX}_{3}$ perovskite NCs, and demonstrate the positive effects and the potential of employing the metal doping/alloying to achieve perovskite NCs-based LEDs with enhanced device efficiency and stability.

\subsection{Green-emissive LEDs based on metal doped/alloyed perovskite NCs}

Following the intense research works on $\mathrm{Mn}^{2+}$ doping of $\mathrm{CsPbX}_{3} \mathrm{NCs}$, Chen and co-workers first investigated the LEDs based $\mathrm{CsPbBr}_{3}$ with a trace amount of $\mathrm{Mn}^{2+}$ substitution. ${ }^{[49]}$ Their calculation results suggested that doping of $\mathrm{Mn}^{2+}$ in $\mathrm{CsPbX}_{3} \mathrm{NCs}$ can significantly enhance the perovskite lattice formation energy, and hence improve the thermal stability and optical performance of the doped NCs. Although there were many reports with high $\mathrm{Mn}^{2+}$ doping ratios in the perovskite NCs, the optimal doping concentration of $\mathrm{Mn}^{2+}$ for achieving the most thermodynamically stable NCs was theoretically predicated to be $\sim 2.08 \mathrm{~mol} \%$ here. The experimental results demonstrated a much more stable PL emission from Mn-doped $\mathrm{CsPBr}_{3}$ solution with an optimal doping ratio of 2.6\%, while undoped and doped NCs with a higher ratio (4.3\%) both exhibited rapid degradation in the PL brightness, when exposed to ambient air or UV light illumination conditions. Thermal stability of Mn-doped $\mathrm{CsPbBr}_{3}$ NCs was also investigated. Although the solution stability is not excellent, the 
PL emission intensities of NCs with $4.3 \% \mathrm{Mn}^{2+}$ doping exhibited much better thermal stability, with $120 \%$ of their initial value retained after three heating and cooling recycling at a high temperature up to $200{ }^{\circ} \mathrm{C}$. Owing to the much-enhanced material properties, the obtained LEDs based on Mndoped $\mathrm{CsPbBr}_{3} \mathrm{NCs}$ exhibit much improved maximum luminance of $9971 \mathrm{~cd} \mathrm{~m}^{-2}$, as compared to that based on undoped ones. ${ }^{[49]}$

Similarly, introducing heterovalent $\mathrm{Ce}^{3+}$ ions into the $\mathrm{CsPbr}_{3}$ NCs can also maintain the integrity of the perovskite structure without generating additional trap states, resulted from the easier formation of higher energy level of conduction band between $\mathrm{Ce}^{3+}$ and bromine. ${ }^{[83]}$ The obtained Ce-doped $\mathrm{CsPbBr}_{3}$ NCs exhibited a significantly increased PLQY up to 89\%, which is two factors higher than the undoped ones. They fabricated LEDs with a device structure of ITO/PEDOT:PSS/Poly-TPD/Ce:CsPbBr 3 /TPBi/LiF/Al (Figure 6a) (wherein, PEDOT:PSS is poly(3,4-ethylenedioxythiophene):polystyrene sulfonate; Poly-TPD is Poly(4-butylphenyldiphenyl-amine), TPBi is 2,2',2"-(1,3,5-Benzinetriyl)-tris(1-phenyl-1-H-benzimidazole); LiF is lithium fluoride., and demonstrated a peak EQE of $1.1 \%$ ( luminance $\approx 1500 \mathrm{~cd} / \mathrm{m}^{2}$ ) and $4.4 \%$ (luminance $\approx 3500 \mathrm{~cd} / \mathrm{m}^{2}$ ) based on the undoped and doped NCs, respectively (Figure 6b and 6c). ${ }^{[83]}$ In addition, the observation of a lower turn voltage $(\approx 2.5 \mathrm{~V})$ for Ce-doped LEDs as compared to undoped (2.8 V) indicates a reduced charge injection barrier in the resulting LEDs.

In addition to the directly doping the NCs during the synthesis, Song et al. recently demonstrated that post treatments of the as-synthesized NCs with metal-halide salts were also efficient to passivate the surface defects, leading to to obvious enhancement in the optoelectronic properties of the obtained NCs and the resulting device performance. ${ }^{[84]}$ Several different metal bromides including $\mathrm{ZnBr}_{2}, \mathrm{MnBr}_{2}, \mathrm{GaBr}_{3}$, and $\mathrm{InBr}_{3}$ were introduced as the inorganic ligands of the obtained NCs. Unlike the previous reported efficient doping, the employed post treatments here are more likely to replace the long-chain organic ligands on the surface of the NCs, and hence led to enhanced PL emission and improved charger transport 
properties. Several optical characterizations and surface sensitive XPS measurements were conducted to confirm the existence of the introduced inorganic ligands only at the NC surface. They observed obviously enhanced PLQY from 58\% to 79\% after the incorporation of $\mathrm{ZnBr}_{2}$, suggesting efficient passivation of the surface defects and enhanced radiative recombination of the treated NCs. In addition, the thermal stability of the PLQY of NCs with inorganic halides post treatments was also improved, owing to the reduced formation of nonradiative recombination centers under elevated temperatures. More importantly, based on the characterizations of single charge carrier devices, the charge transport properties of the post treated NCs were also obviously enhanced. They obtained green-emissive LEDs based on the NCs exhibited much higher EQE and luminance, showing a record high peak EQE of $16.48 \%$ for devices based on $\mathrm{ZnBr}_{2}$ treated NCs, and a record high luminance of over 100,080 cd m $\mathrm{m}^{-2}$ for devices based on $\mathrm{MnBr}_{2}$ treated NCs. ${ }^{[84]}$

\subsection{Red-emissive LEDs based on metal doped/alloyed perovskite NCs}

Incorporation of dopants into the red-emissive perovskite NCs for LEDs with improved device performance was first noticed by Rogach and co-workers, when they characterized the redemissive LEDs fabricated on Ag and indium tin oxide (ITO) substrates. ${ }^{[70]}$ According to the characterization results of the TEM, elemental mapping and elemental analysis, they confirmed the diffusion of $\mathrm{Ag}^{+}$ions into the perovskite NCs films for devices fabricated on Ag substrates. The diffused $\mathrm{Ag}^{+}$ions efficiently passivated the NC surface and converted the nonradiative trap states to radiative ones, leading to enhanced optical and charge transport properties of the redemissive $\mathrm{CsPbI}_{3}$ films. The obtained LEDs on Ag substrates showed a much-increased average peak EQE of $11.2 \%$ compared to the ITO-ones, while the latter shows an average peak EQE of $7.3 \%{ }^{[70]}$

The well investigated isovalent doping of $\mathrm{CsPbI}_{3}$ perovskite NCs using $\mathrm{Mn}^{2+}$ and $\mathrm{Zn}^{2+}$ were also reported to be efficient methods for the fabrication of red-emissive LEDs with improved 
device performance. ${ }^{[49,65]}$ For example, Zou et al. first demonstrated obvious increased EQE of 1.04\% for LEDs based on Mn-doped $\mathrm{CsPbI}_{3}$ NCs. ${ }^{[49]}$ Recently, Shen et al. introduced $\mathrm{Zn}^{2+}$ doping into $\mathrm{CsPbI}_{3} \mathrm{NCs}$ and observed significantly suppressed non-radiative recombination and close-to-unity PLQY of 98.5\%. ${ }^{[65]}$ The space-charge-limited current characterization results revealed almost an order of magnitude decrease of the defect density in $\mathrm{CsPb}_{0.64} \mathrm{Zn}_{0.36} \mathrm{I}_{3}$ NCs, as compared to the undoped ones. The Fermi level was shifted to $-4.43 \mathrm{eV}$ from $-4.17 \mathrm{eV}$ after the $\mathrm{Zn}^{2+}$ doping from the ultraviolet photoelectron spectra (UPS) characterization results. Considering the unchanged conduction band minimum position, the shift in Fermi level indicates that the perovskite NCs switch from n-type to nearly ambipolar nature after sufficient $\mathrm{Zn}^{2+}$ alloying, which is consistent with the observed more balanced electron and hole mobility. They fabricated efficient red-emissive LEDs based on a device structure (Figure 6d) of ITO/ZnO/PEI/Zn-CsPbI $3 / \mathrm{TCTA} / \mathrm{Mo}_{3} / \mathrm{Au}$ (wherein, PEI is Polyethylenimine, TCTA is 4,4',4"Tris(carbazol-9-yl)triphenylamine) and demonstrated a peak EQE of 15.1\% for devices based on Zn-doped NCs, along with improved maximum luminance of $2202 \mathrm{~cd} \mathrm{~m}^{-2}$ (Figure 6e and 6f). ${ }^{[65]}$ In addition, due to the smaller radius of $\mathrm{Zn}^{2+}$ ions, Goldschmidt tolerance factors of the obtained $\mathrm{Zn}$-doped NCs were pushed to a more stable region with increasing $\mathrm{Zn}^{2+}$ doping ratio, leading to further enhanced stability of the $\mathrm{Zn}$-alloyed $\mathrm{CsPbI}_{3} \mathrm{NCs}$, which is expected to further improve the device stability of the resulting LEDs.

The divalent doping strategy of $\mathrm{CsPbI}_{3}$ NCs can be combined with efficient halide surface passivation to further improve the material properties and the device performance. Yao et al. ${ }^{[85]}$ and Lu et al. ${ }^{[86]}$ independently demonstrated high performance red-emissive LEDs based on $\mathrm{CsPbI}_{3} \mathrm{NCs}$ with simultaneous strontium $\left(\mathrm{Sr}^{2+}\right)$ doping and iodide/chlorine surface passivation. Owing to the slightly smaller ion radius of $\mathrm{Sr}^{2+}(1.18 \AA)$ than the $\mathrm{Pb}^{2+}(1.19 \AA)$ ions, doping of the $\mathrm{Sr}^{2+}$ into the $\mathrm{CsPbI}_{3} \mathrm{NCs}$ may cause a slight lattice contraction. At the same time, halide anions of $\mathrm{I}^{-}$and $\mathrm{Cl}^{-}$can efficiently passivate the defects at the surface of NCs, resulting in 
improved radiative recombination rates in the treated NCs. Yao et al. demonstrated that stable cubic-phase $\mathrm{CsPbI}_{3}$ with tunable size from 15 to sub- $5 \mathrm{~nm}$ through introducing $\mathrm{Sr}^{2+}$ substitution along with iodide passivation. ${ }^{[85]}$ It was found that the incorporation of $\mathrm{Sr}^{2+}$ ions significantly increased the formation energies of the perovskite structure and reduced the structure distortion for small-sized $\mathrm{CsPbI}_{3}$ NCs. The obtained few-nanometer-sized doped $\mathrm{CsPbI}_{3} \mathrm{NCs}$ still retained the high PL emission properties and highly close packing in the deposited thin films, with improved PL stability of the colloidal solution and the thin films. The obtained devices based on $3.1 \% \mathrm{Sr}^{2+}$ doped $\mathrm{CsPbI}_{3} \mathrm{NCs}(5.38 \mathrm{~nm})$ exhibited a high EQE of $5.92 \% .{ }^{[85]} \mathrm{In}$ addition, due to the improved material stability, the resulting LEDs based on $\mathrm{Sr}^{2+}$-substituted $\mathrm{CsPbI}_{3} \mathrm{NCs}$ exhibited a $L_{50}$ (needed time decay to $50 \%$ of the initial luminance) of 120 min, which is significantly improved as compared to the devices based on undoped ones (10 min). Simultaneously introducing $\mathrm{Sr}^{2+}$ doping and $\mathrm{Cl}^{-}$surface passivation can also generate a high PLQY of $84 \%$ for $\mathrm{CsPbI}_{3} \mathrm{NCs}$ with further enhanced materials stability. The resulting devices based on $\mathrm{CsPbI}_{3} \mathrm{NCs}$ with $10 \% \mathrm{Sr}^{2+}$ substitution exhibit a much higher EQE of $13.5 \% .{ }^{[86]}$

\subsection{Blue-emissive LEDs based on metal doped/alloyed perovskite NCs}

Unlike the great success and fast improvement in the green- and red-emissive LEDs, the blueemissive devices based on perovskite NCs, especially the pure blue ones that meet the National Television System Committee (NTSC) standard, still exhibit low device efficiencies. ${ }^{[87]}$ Through carefully tuning the $\mathrm{Mn}^{2+}$ doping concentration at a lower concentration with a modified synthesis, Hou et al. found that the blue emission from of $\mathrm{Mn}$-doped $\mathrm{CsPbCl}_{3}$ can be significantly enhanced, without introducing obvious $\mathrm{Mn}^{2+}$ dopant emission. ${ }^{88}$ ] It was demonstrated in early works that the $\mathrm{Mn}^{2+}$ dopant emission can be efficiently suppressed using a bromide-rich synthesis process. ${ }^{[45]}$ The targeted blue perovskite emission in the range of 467$470 \mathrm{~nm}$ was achieved through a two-step sequential synthesis approach. They first synthesized the Cl-rich NCs with high $\mathrm{Mn}^{2+}$ doping concentration by hot injection method and then 
exchanged the obtained NCs with $\mathrm{Br}^{-}$at room temperature to the desired PL emission. It was found that the sequential synthesis can increase the Mn-doping from $0.09 \%$ to $1.5 \%$ without decreasing the strong blue emission at $468 \mathrm{~nm}$. The controllable Mn-doping (0.19\%) gave a three times higher PLQY of 28\% than the undoped ones. ${ }^{[88]}$ Further increasing the $\mathrm{Mn}^{2+}$ doping ratio decreased PLQY and resulted in the appearance of more obvious $\mathrm{Mn}^{2+}$ dopant emission. Interestingly, no Mn dopant emission was observed in the fabricated device even with a high doping ratio of $1.5 \%$, which was ascribed to the saturated emission originating from the long emissive lifetime or the top casted TFB (Poly(9,9-dioctylfluorene-alt-N-(4-sec-butylphenyl)diphenylamine)) hole transport layer. They were employed to fabricate LEDs using ITO/PEDOT:PSS/TFB/PFI/Mn:CsPbBr ${ }_{3-\mathrm{x}} \mathrm{Cl}_{\mathrm{x}} / \mathrm{TPBi} / \mathrm{LiF} / \mathrm{Al}$ (wherein, PFI is perfluorinated ionomer) device structure (Figure 6g). The optimized devices based on a 0.19\% Mn doping exhibited a 4-fold improvement in the EQE from 0.5\% to 2.12\% (Figure 6h and 6i), which represents the highest efficiency for pure blue LEDs based on perovskite NCs. ${ }^{[8]}$ 

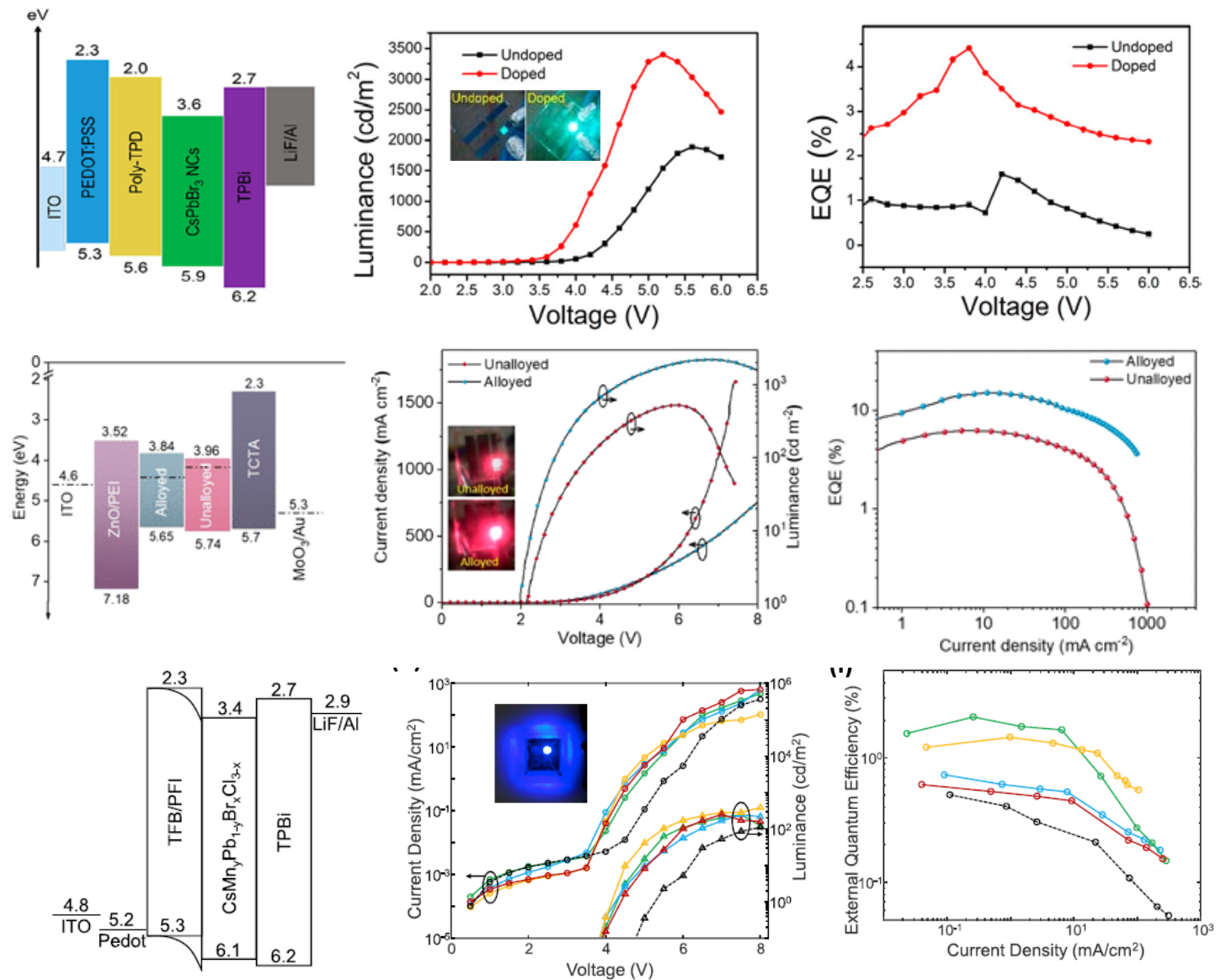

Figure 6. Device structure and J-V-L (current density-voltage-luminance) characteristics of metal doped perovskite NC-LEDs: (a) Device structure of Ce-doped CsPbBr3 $\mathrm{NC}_{\mathrm{LED}}$ (b) Luminance vs applied voltage characteristics, inset photograph of LED. (c) EQE vs applied voltage. Reproduced with permission. ${ }^{[83]}$ Copyright (C) 2018, American Chemical Society. (d) Device structure of Zn-doped $\mathrm{CsPbI}_{3}$ NC-LED and (e) J-V-L characteristics, inset photograph of NC-LED (f) EQE vs current density. Reproduced with permission. ${ }^{[65]}$ Copyright (C) 2019 American Chemical Society. (g) Device structure of Mn-doped NC-LED (h) J-V-L characteristics, inset photograph of NC-LED (i) EQE vs current density with $\mathrm{Mn}^{2+}$ doping concentration. Reproduced with permission. ${ }^{[88]}$ Copyright $@ 2018$ Elsevier Inc. 
Table 1 Summary of performance parameters of metal doped green, red, and blue emissive perovskite NC LEDs.

\begin{tabular}{|c|c|c|c|c|}
\hline Device structure & $\begin{array}{l}\text { PLQYa } \\
(\%)\end{array}$ & $\begin{array}{l}E L^{b} \\
(\mathbf{n m})\end{array}$ & $\begin{array}{l}\text { Luminance }^{c} \\
(\mathrm{~cd} / \mathrm{m} 2)\end{array}$ & $\begin{array}{l}\text { EQE }^{\mathrm{d}} \\
(\%)\end{array}$ \\
\hline ITO/PEDOT:PSS/PolyTPD/Mn:CsPbBr $3 / \mathrm{TPBi} / \mathrm{LiF} / \mathrm{Al}$ & - & 512 & 9971 & $1.49^{[49]}$ \\
\hline ITO/PEDOT:PSS/PolyTPD/Ce:CsPbBr 3 /TPBi/LiF/Al & 89 & 520 & 3500 & $4.4^{[83]}$ \\
\hline ITO/PEDOT:PSS/PTAA/Zn:CsPbBr/ $/ \mathrm{TPBi} / \mathrm{LiF} / \mathrm{Al}$ & 76 & 518 & 76940 & $16.4^{[84]}$ \\
\hline ITO/PEDOT:PSS/PTAA/Mn:CsPbBr 3 /TPBi/LiF/Al & 79 & - & 100080 & $15.6^{[84]}$ \\
\hline ITO/PEDOT:PSS/PTAA/Ga:CsPbBr 3 /TPBi/LiF/Al & 74 & - & 53600 & $14.1^{[84]}$ \\
\hline ITO/PEDOT:PSS/PTAA/In:CsPbBr 3 /TPBi/LiF/Al & 77 & - & 76200 & $16.2^{[84]}$ \\
\hline $\mathrm{ITO} / \mathrm{Ag} / \mathrm{ZnO} / \mathrm{PEI} / \mathrm{CsPbI}_{3} / \mathrm{TCTA} / \mathrm{MoO}_{3} / \mathrm{Au}$ & $>60$ & 690 & 1106 & $11.2^{[70]}$ \\
\hline ITO/PEDOT:PSS/PolyTPD/Mn:CsPbI 3 /TPBi/LiF/Al & 89 & 685 & 132 & $1.04^{[51]}$ \\
\hline ITO/ZnO/PEI/Zn:CsPbI ${ }_{3} / \mathrm{TCTA} / \mathrm{MoO}_{3} / \mathrm{Au}$ & 98.5 & 690 & 2202 & $15.1^{[65]}$ \\
\hline ITO/PEDOT:PSS/PolyTPD/Sr:CsPbBr 3 /TPBi/LiF/Al & $>80$ & 678 & 1250 & $5.92^{[85]}$ \\
\hline ITO/ZnO/PEI/Sr:CsPbI ${ }_{3} / \mathrm{TCTA} / \mathrm{MoO}_{3} / \mathrm{Au}$ & 84 & 691 & 1152 & $13.5^{[86]}$ \\
\hline $\begin{array}{l}\text { ITO/PEDOT:PSS/TFB/PFI/Mn:CsPbBr }{ }_{3-} \\
{ }_{x} \mathrm{Cl}_{x} / \mathrm{TPBi} / \mathrm{LiF} / \mathrm{Al}\end{array}$ & 28 & 464 & 245 & $2.12^{[88]}$ \\
\hline
\end{tabular}

${ }^{\mathrm{a}}$ Maximum PL QY of metal doped perovskite NCs, ${ }^{\mathrm{b}}$ Electroluminescence (EL) peak, Maximum ${ }^{\mathrm{c}}$ uminance, ${ }^{\mathrm{d}}$ external quantum efficiency of metal doped PeLEDs.

\section{Summary and Outlook}

In this review, we have summarized recent progresses of metal doping/alloying in all-inorganic $\mathrm{CsPbX}_{3}$ perovskite NCs with various doping strategies and also their applications in LEDs with different emission colours. We note that further investigations on the metal doped/alloyed perovskite NCs would be beneficial to achieve efficient control over the materials properties of the final products. For example, detailed characterizations on the existing status of the metal dopants in the final products and the effects on the structural properties, especially the change of the crystal lattice and the crystal stability of the perovskite NCs are still needed. In addition, an in-depth understanding of the doping/alloying mechanism and detailed characterizations on the photophysical properties of the doped perovskite NCs are also required to provide insights for future doping strategies. Moreover, exploration of more efficient metal dopants or developing co-doping strategies could further improve the optoelectronic properties and the materials stability of perovskite NCs, leading to enhanced device efficiency and stability of the 
ensuing optoelectronic devices. Although the research on the metal doping/alloying of perovskite NCs is still at an early stage, it provides a new direction to achieve perovskite NCs with improved materials properties and stability, which should be useful for the fabrication of high-performance LEDs and also other optoelectronic devices based on perovskite NCs.

\section{Acknowledgements}

This work is supported by the ERC Starting Grant (717026),and the European Commission Marie Skłodowska-Curie Actions (691210), and the Swedish Government Strategic Research Area in Materials Science on Functional Materials at Linköping University (Faculty Grant SFOMat-LiU no. 2009-00971). Z.C. Yuan thanks the financial support from the China Scholarship Council.

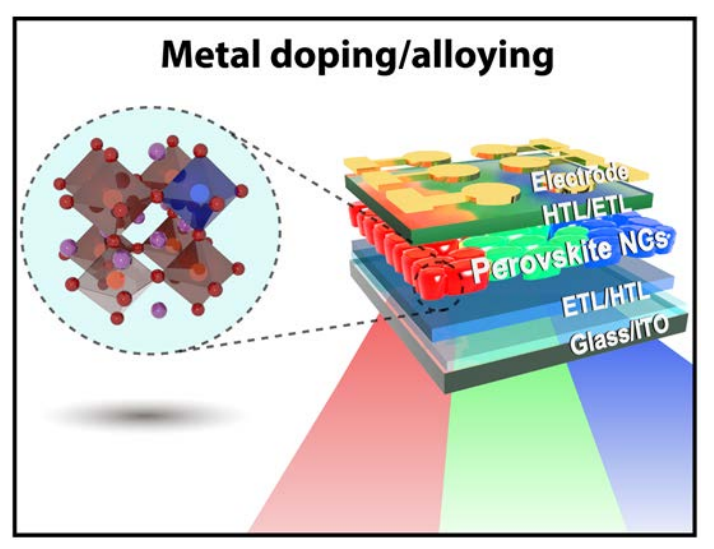

ToC
Naresh Kumar Kumawat, Zhongcheng Yuan, Sai Bai*, and Feng Gao*

Metal doping/alloying of cesium lead halide perovskite nanocrystals and their applications in light-emitting diodes with enhanced efficiency and stability 


\section{References}

[1] (a) D. Weber, Z. Naturforsch. 1978, 33 b, 1443-1445. (b) G.C. Papavassiliou, I.R. Koutselas, Synthetic Metals 1995, 71, 1713-1714. (c) K. Tanakaa, T. Takahashia, T. Bana, T. Kondo, K. Uchidab, N. Miurab, Solid State Communications 2003, 127, 619-623.

[2] I. E. Castelli, J. M. Garcia-Lastra, K. S. Thygesen, K. W. Jacobsen, APL Mater. 2014, 2, 081514.

[3] S. D. Stranks, G. E. Eperon, G. Grancini, C. Menelaou, M. J. P. Alcocer, T. Leijtens, L. M. Herz, A. Petrozza, H. J. Snaith, Science 2013, 342, 341-344.

[4] T. Leijtens, S. D. Stranks, G. E. Eperon, R. Lindblad, E. M. J. Johansson, I. J. McPherson, H. Rensmo, J. M. Ball, M. M. Lee, H. J. Snaith, ACS Nano 2014, 8, 7147-7155.

[5] (a) F. Li, C. Ma, H. Wang, W. Hu, W. Yu, A. D. Sheikh, Tom Wu, Nat. Commun. 2015, 6, 8238. (b) N. K. Kumawat, D. Gupta, D. Kabra, Energy Technol. 2017, 5, 1734-1749.

[6] C. K. Moller. Nature 1958, 182, 1436.

[7] N. Kitazawa, Y. Watanabe, Y. nakamura, Journal of Materials Science 2002, 37, 35853587.

[8] N. J. Jeon, J. H. Noh, W. S. Yang, Y. C. Kim, S. Ryu, J. Seo, S. Il Seok, Nature 2015, 517, 476-480.

[9] J. H. Noh, S. H. Im, J. H. Heo, T. N. Mandal, S. Il Seok, Nano Lett. 2013, 13, 1764-1769.

[10] N. K. Kumawat, A. Dey, K. L. Narasimhan, D. Kabra, ACS Photonics 2015, 2, 349-354.

[11] V. Prakasam, F. D. Giacomo, R. Abbel, D. Tordera, M. Sessolo, G. Gelinck, H. J. Bolink, ACS Appl. Mater. Interfaces 2018, 10, 41586-41591.

[12] (a) R. Comin, G. Walters, E. S. Thibau, O. Voznyy, Z.-H. Lub, E. H. Sargent, J. Mater. Chem. C 2015, 3, 8839-8843. (b) N. K. Kumawat, M. N. Tripathi, U. Waghmare, D. Kabra, J. Phys. Chem. A 2016, 120, 3917-3923.

[13] (a) A. Kojima, K. Teshima, Y. Shirai, T. Miyasaka, J. Am. Chem. Soc. 2009, 131, 60506051. (b) Q. Zhang, R. Su, W. Du, X. Liu, L. Zhao, S. T. Ha, Q. Xiong, Small Methods 2017, $1,1700163$.

[14] Z.-K. Tan, R. S. Moghaddam, M. L. Lai, P. Docampo, R. Higler, F. Deschler, M. Price, A. Sadhanala, L. M. Pazos, D. Credgington, F. Hanusch, T. Bein, H. J. Snaith, R. H. Friend, Nature Nanotechnology 2014, 9, 687-692.

[15] N. K. Kumawat, A. Dey, K. L. Narshimhan, D. Kabra, ACS Appl. Mater. Interfaces 2015, 7, 13119-13124.

[16] (a) C. Bao, J. Yang, S. Bai, W. Xu, Z. Yan, Q. Xu, J. Liu, W. Zhang, Feng Gao, Adv. Mater. 2018, 30, 1803422. (b) M. Ahmadi, Ting Wu, Bin Hu, Adv. Mater. 2017, 29, 1605242. 
[17] L. C. Schmidt, A. Pertegas, S. Gonzalez-Carrero, O. Malinkiewicz, S. Agouram, G. M. Espallargas, H. J. Bolink, R. E. Galian, J. Perez-Prieto, J. Am. Chem. Soc. 2014, 136, 850-853.

[18] F. Zhang, H. Zhong, C. Chen, X. G. Wu, X. Hu, H. Huang, J. Han, B. Zou, Y. Dong, ACS Nano 2015, 9, 4533-4542.

[19] L. Protesescu, S. Yakunin, M. I. Bodnarchuk, F. Krieg, R. Caputo, C. H. Hendon, R. X. Yang, A. Walsh, M. V. Kovalenko, Nano Lett. 2015, 15, 3692-3696.

[20] J. A. Sichert, Y. Tong, N. Mutz, M. Vollmer, S. Fischer, K. Z. Milowska, R. Garcia Cortadella, B. Nickel, C. Cardenas-Daw, J. K. Stolarczyk, A. S. Urban, J. Feldmann, Nano Lett. 2015, 15, 6521-6527.

[21] M. Kulbak, S. Gupta, N. Kedem, I. Levine, T. Bendikov, G. Hodes, D. Cahen, J. Phys. Chem. Lett. 2016, 7, 167-172.

[22] X. L. Zhang, B. Xu, J. B. Zhang, Y. Gao, Y. J. Zheng, K. Wang, X. W. Sun, Adv. Funct. Mater. 2016, 26, 4595-4600.

[23] P. Ramasamy, D.-H. Lim, B. Kim, S.-H. Lee, M.-S. Leeb, J.-S. Lee, Chem. Commun. 2016, 52, 2067-2070.

[24] A. Swarnkar, A. R. Marshall, E. M. Sanehira, B. D. Chernomordik, D. T. Moore, J. A. Christians, T. Chakrabarti, J. M. Luther, Science 2016, 354, 92-95.

[25] Y. Wang, X. Li, X. Zhao, L. Xiao, H. Zeng, H. Sun, Nano Lett. 2016, 16, 448-453.

[26] S. S. Mali, C. S. Shim, C. K. Hong, NPG Asia Mater. 2015, 7, 208.

[27] J. Song, J. Li, X. Li, L. Xu, Y. Dong, H. Zeng, Adv. Mater. 2015, 27, 7162-7167.

[28] Y. Dong , Y. Gu , Y. Zou , J. Song, L. Xu , J. Li , J. Xue , X. Li, H. Zeng, small 2016,12, 5622-5632.

[29] J. Song, J. Li, L. Xu, J. Li, F. Zhang, B. Han, Q. Shan, H. Zeng, Adv. Mater. 2018, 30, 1800764.

[30] J. Li, L. Xu, T. Wang, J. Song, J. Chen, J. Xue, Y. Dong, B. Cai, Q. Shan, B. Han, H. Zeng, Adv. Mater. 2017, 29, 1603885.

[31] S. Bai, Z. Yuan, F. Gao, J. Mater. Chem. C, 2016, 4, 3898-3904.

[32] Y. Dong, Y. Zhao, S. Zhang, Y. Dai, L. Liu, Y. Li, Q. Chen, J. Mater. Chem. A 2018, 6, 21729-21746.

[33] Q. A. Akkerman, G. Raino, M. V. Kovalenko, L. Manna, Nature Materials 2018, 17, 394405.

[34] J. Shamsi, A. S. Urban, M. Imran, L. D. Trizio, L. Manna, Chem. Rev. 2019; DOI: 10.1021/acs.chemrev.8b00644.

[35] Q. Zhang, Y. Yin, ACS Cent. Sci. 2018, 4, 668-679. 
[36] X. Chen, F. Zhang, Y. Ge, L. Shi, S. Huang, J. Tang, Z. Lv, L. Zhang, B. Zou, H. Zhong, Adv. Funct. Mater. 2018, 28, 1706567.

[37] F. Fang, W. Chen, Y. Li, H. Liu, M. Mei, R. Zhang, J. Hao, M. Mikita, W. Cao, R. Pan, K. Wang, X. W. Sun, Adv. Funct. Mater. 2018, 28, 1706000.

[38] E. Yassitepe, Z. Yang, O. Voznyy, Y. Kim, G. Walters, J. A. Castaneda, P. Kanjanaboos, M. Yuan, X. Gong, F. Fan, J. Pan, S. Hoogland, R. Comin, O. M. Bakr, L. A. Padilha, A. F. Nogueira, E. H. Sargent, Adv. Funct. Mater. 2016, 26, 8757-8763.

[39] L. Wu, Q. Zhong, D. Yang, M. Chen, H. Hu, Q. Pan, H. Liu, M. Cao, Y. Xu, B. Sun, Q. Zhang, Langmuir 2017, 33, 12689-12696.

[40] Binbin Luo, S. B. Naghadeh, J. Z. Zhang, ChemNanoMat 2017, 3, 456 - 465

[41] (a) A. Pan, B. He, X. Fan, Z. Liu, J. J. Urban, A. P. Alivisatos, L. He, Yi Liu, ACS Nano 2016, 10, 7943-7954. (b) N. K. Kumawat, A. Swarnkar, A. Nag, D. Kabra, J. Phys. Chem. C 2018, 122, 13767-13773.

[42] D. Yang, X. Li, H. Zeng, Adv. Mater. Interfaces 2018, 5, 1701662.

[43] J. H. Park, Ah-young Lee, J. C. Yu, Y. S. Nam, Y. Choi, J. Park, M. H. Song, ACS Appl. Mater. Interfaces 2019, 11, 8428-8435.

[44] D. Parobek, B. J. Roman, Y. Dong, H. Jin, E. Lee, M. Sheldon, D. H. Son, Nano Lett. 2016, 16, 7376-7380.

[45] W. Liu, Q. Lin, H. Li, K. Wu, I. Robel, J. M. Pietryga, V. I. Klimov, J. Am. Chem. Soc. 2016, 138, 14954-14961.

[46] H. Liu, Z. Wu, J. Shao, D. Yao, H. Gao, Y Liu, W. Yu, H. Zhang, B. Yang, ACS Nano 2017, 11, 2239-2247.

[47] D. Chen, G. Fang, X. Chen, L. Lei, J. Zhong, Q. Mao, S. Zhoub, J. Lib, J. Mater. Chem. C 2018, 6, 8990-8998.

[48] W. Chen, T. Shi, J. Du, Z. Zang, Z. Yao, M. Li, K. Sun, W. Hu, Y. Leng, X. Tang, ACS Appl. Mater. Interfaces 2018, 10, 43978-43986.

[49] S. Zou, Y. Liu, J. Li, C. Liu, R. Feng, F. Jiang, Y. Li, J. Song, H. Zeng, M. Hong, X. Chen, J. Am. Chem. Soc. 2017, 139, 11443-11450.

[50] X. Li, Y. Guo, B. Luo, Crystals 2018, 8, 4; DOI:10.3390/cryst8010004.

[51] X. Li, Y. Wu, S. Zhang, B. Cai, Y. Gu, J. Song, H. Zeng, Adv. Funct. Mater. 2016, 26, $2435-2445$.

[52] X. Du, G. Wu, J. Cheng, H. Dang, K. Ma, Y.-W. Zhang, P.-F. Tana, S. Chen, RSC Adv. 2017, 7, 10391-10396. 
[53] F. Krieg, S. T. Ochsenbein, S. Yakunin, S ten Brinck, P. Aellen, A. Suess, B. Clerc, D. Guggisberg, O. Nazarenko, Y. Shynkarenko, S. Kumar, C.-J. Shih, I. Infante, M. V. Kovalenko, ACS Energy Lett. 2018, 3, 641-646.

[54] G. Nedelcu, L. Protesescu, S. Yakunin, M. I. Bodnarchuk, M. J. Grotevent, M. V. Kovalenko, Nano Lett. 2015, 15, 5635-5640.

[55] Q. A. Akkerman, V. D’Innocenzo, S. Accornero, A. Scarpellini, A. Petrozza, M. Prato, L. Manna, J. Am. Chem. Soc. 2015, 137, 10276-10281.

[56] J.-P. Correa-Baena, A. Abate, M. Saliba, W. Tress, T. J. Jacobsson, M. Gratzelc, Anders Hagfeldt, Energy Environ. Sci. 2017, 10, 710-727.

[57] T. J. Jacobsson, J.-P. Correa-Baena, M. Pazoki, M. Saliba, K. Schenk, M. Gratzel, A. Hagfeldt, Energy Environ. Sci., 2016, 9, 1706-1724.

[58] Z. Zhao, W. Xu, G. Pan, Y. Liu, M. Yang, S. Hua, X. Chen, H. Peng, H. Song, Material Research Bulletin 2018, 112, 142-146.

[59] D. Amgar, T. Binyamin, V. Uvarov, L. Etgar, Nanoscale 2018, 10, 6060-6068.

[60] M. R. Linaburg, E. T. McClure, J. D. Majher, P. M. Woodward, Chem. Mater. 2017 29, 3507-3514.

[61] X. Yuan, S. Ji, M. C. De Siena, L. Fei, Z. Zhao, Y. Wang, H. Li, J. Zhao, D. R. Gamelin, Chem. Mater. 2017, 29, 8003-8011.

[62] J. Zhu, X. Yang, Y. Zhu, Y. Wang, J. Cai, J. Shen, L. Sun, C. Li, J. Phys. Chem. Lett. 2017, 8, 4167-4171.

[63] A. K. Guria, S. K. Dutta, S. D. Adhikari and N. Pradhan, ACS Energy Lett. 2017, 2, 1014 1021.

[64] W. van der Stam, J. J. Geuchies, T Altantzis, K. H. W. van den Bos, J. D. Meeldijk, S. Van Aert, S. Bals, D. Vanmaekelbergh, C. de Mello Donega, J. Am. Chem. Soc. 2017, 139, 40874097.

[65] X. Shen, Y. Zhang, S. V. Kershaw, T. Li, C. Wang, X. Zhang, W. Wang, D. Li, Y. Wang, Min Lu, L. Zhang, C. Sun, D. Zhao, G. Qin, X. Bai, W. W. Yu, A. L. Rogach, Nano Lett. 2019; DOI: 10.1021/acs.nanolett.8b04339.

[66] F. Li, Y. Liu, H. Wang, Q. Zhan, Q. Liu, Zhiguo Xia, Chem. Mater. 2018, 30, 8546-8554.

[67] N. Mondal, A. De, A. Samanta, ACS Energy Lett. 2019, 4, 32-39.

[68] C. Bi, S. Wang, Q. Li, S. V. Kershaw, J. Tian, A. L. Rogach, J. Phys. Chem. Lett. 2019, $10,943-952$.

[69] Z.-J. Yong, S.-Q. Guo, J.-P. Ma, J.-Y. Zhang, Z.-Y. Li, Y.-M. Chen, B.-B. Zhang, Y. Zhou, J. Shu, J.-L. Gu, L.-R. Zheng, O. M. Bakr, H.-T. Sun, J. Am. Chem. Soc. 2018, 140, 9942-9951. 
[70] M. Lu, X. Zhang, X. Bai, H. Wu, X. Shen, Y. Zhang, W. Zhang, W. Zheng, H. Song, W. W. Yu, A. L. Rogach, ACS Energy Lett. 2018, 3, 1571-1577.

[71] M. Liu, G. Zhong, Y. Yin, J. Miao, K. Li, C. Wang, X. Xu, C. Shen, H. Meng, Adv. Sci. 2017, 4, 1700335.

[72] R. Begum, M. R. Parida, A. L. Abdelhady, B. Murali, N. M. Alyami, G. H. Ahmed, M. N. Hedhili, O. M. Bakr, O. F. Mohammed, J. Am. Chem. Soc. 2017, 139, 731-737.

[73] S. Zou, G. Yang, T. Yang, D. Zhao, Z. Gan, W. Chen, H. Zhong, X. Wen, B. Jia, B. Zou, J. Phys. Chem. Lett. 2018, 9, 4878-4885.

[74] F.-P. Zhu, Z.-J. Yong, B.-M. Liu, Y.-M. Chen, Y. Zhou, J.-P. Ma, H.-T. Sun, Y.-Z. Fang, Optics Express 2017, 25, 33283-33289.

[75] S. Zhou, Y. Ma, G. Zhou, X. Xu, M. Qin, Y. Li, Y.-J. Hsu, H. Hu, G. Li, N. Zhao, J. Xu, X. Lu, ACS Energy Lett. 2019, 4, 534-541.

[76] G. Pan, X. Bai, D. Yang, X. Chen, P. Jing, S. Qu, L. Zhang, D. Zhou, J. Zhu, W. Xu, B. Dong, H. Song, Nano Lett. 2017, 17, 8005-8011.

[77] D. Zhou, D. Liu, G. Pan, X. Chen, D. Li, W. Xu, X. Bai, H. Song, Adv. Mater. 2017, 29, 1704149.

[78] T. J. Milstein, K. T. Kluherz, D. M. Kroupa, C. S. Erickson, J. J. De Yoreo, D. R. Gamelin, Nano Lett. 2019; DOI: 10.1021/acs.nanolett.8b05104.

[ 79 ] Y. Zou, Z. Yuan, S. Bai, F. Gao, B. Sun, Materials Today Nano 2019; DOI.org/10.1016/j.mtnano.2019.100028.

[80] T. Chiba, Y. Hayashi, H. Ebe, K. Hoshi, J. Sato, S. Sato, Y.-J. Pu, S. Ohisa, J. Kido, Nature Photonics 2018,12, 681-687.

[81] Y.-H. Kima, H. Choa, T.-W. Lee, PNAS 2016, 113, 11694-11702.

[82] M. V. Kovalenko, L. Protesescu, M. I. Bodnarchuk, Science 2017, 358, 745-750.

[83] J.-S. Yao, J. Ge, B.-N. Han, K.-H. Wang, H.-B. Yao, H.-L. Yu, J.-H. Li, B.-S. Zhu, J.-Z. Song, C. Chen, Q. Zhang, H.-B. Zeng, Y. Luo, S.-H. Yu, J. Am. Chem. Soc. 2018, 140, 36263634.

[84] J. Song, T. Fang, J. Li, L. Xu, F. Zhang, B. Han, Q. Shan, H. Zeng, Adv. Mater. 2018, 30, 1805409.

[85] J.-S. Yao, J. Ge, K.-H. Wang, G. Zhang, B.-S. Zhu, C. Chen, Q. Zhang, Y. Luo, S.-H. Yu, H.-B. Yao, J. Am. Chem. Soc. 2019, 141, 2069-2079.

[86] M. Lu, X. Zhang, Y. Zhang, J. Guo, X. Shen, W. W. Yu, A. L. Rogach, Adv. Mater. 2018, 30, 1804691.

[87] N. K. Kumawat, X.-K. Liu, D. Kabra, Feng Gao, Nanoscale 2019, 11, 2109-2120.

[88] S. Hou, M. K. Gangishetty, Q. Quan, D. N. Congreve, Joule 2018, 2, 2421-2433. 